ESAIM: COCV 22 (2016) 1382-1411

DOI: $10.1051 / \mathrm{cocv} / 2016042$
ESAIM: Control, Optimisation and Calculus of Variations

www.esaim-cocv.org

\title{
AN INTERNAL OBSERVABILITY ESTIMATE FOR STOCHASTIC HYPERBOLIC EQUATIONS *
}

\author{
XiaOyu Fu ${ }^{1}$, Xu LiU ${ }^{2}$, Qi L $\ddot{U}^{1}$ AND Xu Zhang ${ }^{1}$
}

\begin{abstract}
This paper is addressed to establishing an internal observability estimate for some linear stochastic hyperbolic equations. The key is to establish a new global Carleman estimate for forward stochastic hyperbolic equations in the $L^{2}$-space. Different from the deterministic case, a delicate analysis on the adaptedness for some stochastic processes is required in the stochastic setting.
\end{abstract}

Mathematics Subject Classification. 93B05, 93B07, 93C20.

Received June 6, 2016. Accepted June 7, 2016.

\section{INTRODUCTION AND MAIN RESULT}

Let $T>0$ and $\left(\Omega, \mathcal{F},\left\{\mathcal{F}_{t}\right\}_{t \geq 0}, \mathbb{P}\right)$ be a complete filtered probability space, on which a one-dimensional standard Brownian motion $\{B(t)\}_{t \geq 0}$ is defined such that $\mathbb{F}=\left\{\mathcal{F}_{t}\right\}_{t \geq 0}$ is the natural filtration generated by $B(\cdot)$, augmented by all the $\mathcal{P}$-null sets in $\mathcal{F}$. Let $\mathcal{H}$ be a Banach space, and let $C([0, T] ; \mathcal{H})$ be the Banach space of all $\mathcal{H}$-valued strongly continuous functions defined on $[0, T]$. We denote by $L_{\mathbb{F}}^{2}(0, T ; \mathcal{H})$ the Banach space consisting of all $\mathcal{H}$-valued $\mathbb{F}$-adapted processes $X(\cdot)$ such that $\mathbb{E}\left(|X(\cdot)|_{L^{2}(0, T ; \mathcal{H})}^{2}\right)<\infty$, with the canonical norm; by $L_{\mathbb{F}}^{\infty}(0, T ; \mathcal{H})$ the Banach space consisting of all $\mathcal{H}$-valued $\mathbb{F}$-adapted essentially bounded processes; and by $L_{\mathbb{F}}^{2}(\Omega ; C([0, T] ; \mathcal{H}))$ the Banach space consisting of all $\mathcal{H}$-valued $\mathbb{F}$-adapted continuous processes $X(\cdot)$ such that $\mathbb{E}\left(|X(\cdot)|_{C([0, T] ; \mathcal{H})}^{2}\right)<\infty$. Similarly, one can define $L_{\mathbb{F}}^{\infty}\left(\Omega ; C^{m}([0, T] ; \mathcal{H})\right)$ for any positive integer $m$.

Let $G \subset \mathbb{R}^{n}$ (for some $n \in \mathbb{N}$ ) be a nonempty bounded domain with a $C^{2}$ boundary $\Gamma$. Set $Q=(0, T) \times G$ and $\Sigma=(0, T) \times \Gamma$. Assume that $b^{i j} \in C^{2}(\bar{G})(i, j=1,2, \ldots, n)$ satisfy

$$
b^{i j}(x)=b^{j i}(x), \quad \forall x \in \bar{G},
$$

\footnotetext{
Keywords and phrases. Stochastic hyperbolic equation, observability estimate, global Carleman estimate, adaptedness, optimal control.

* This work is partially supported by the NSF of China under grants 11231007, 11322110, 11371084 and 11471231, by the Fundamental Research Funds for the Central Universities under grants 2412015BJ011 and 2015SCU04A02, by the Foundation for the Author of National Excellent Doctoral Dissertation of China under grant 201213, by the Program for New Century Excellent Talents in University under grant NCET-12-0812, by the Fok Ying Tong Education Foundation under grant 141001, and by the Chang Jiang Scholars Program from Chinese Education Ministry. The authors highly appreciate the anonymous referees for their constructive comments which led to this improved version.

1 School of Mathematics, Sichuan University, Chengdu 610064, P.R. China. xiaoyufu@scu.edu.cn; lu@scu.edu.cn; zhang_xu@scu.edu.cn

2 School of Mathematics and Statistics, Northeast Normal University, Changchun 130024, P.R. China. liux216@nenu.edu.cn
} 
and for some constant $s_{0}>0$,

$$
\sum_{i, j=1}^{n} b^{i j}(x) \xi^{i} \xi^{j} \geq s_{0}|\xi|^{2}, \quad \forall(x, \xi)=\left(x, \xi^{1}, \ldots, \xi^{n}\right) \in \bar{G} \times \mathbb{R}^{n} .
$$

Let us consider the following stochastic hyperbolic equation:

$$
\begin{cases}\mathrm{d} y_{t}-\sum_{i, j=1}^{n}\left(b^{i j} y_{x_{i}}\right)_{x_{j}} \mathrm{~d} t=\left(b_{1} y+f\right) \mathrm{d} t+\left(b_{2} y+g\right) \mathrm{d} B(t) & \text { in } Q, \\ y=0 & \text { on } \Sigma, \\ y(0)=y_{0}, y_{t}(0)=y_{1} & \text { in } G,\end{cases}
$$

where $\left(y_{0}, y_{1}\right) \in L^{2}(G) \times H^{-1}(G), f, g \in L_{\mathbb{F}}^{2}\left(0, T ; L^{2}(G)\right)$,

$$
b_{1} \in L_{\mathbb{F}}^{\infty}\left(0, T ; L^{p}(G)\right) \text { with } p \in[n, \infty] \quad \text { and } \quad b_{2} \in L_{\mathbb{F}}^{\infty}\left(0, T ; L^{\infty}(G)\right) .
$$

Also, set

$$
H_{T}=L_{\mathbb{F}}^{2}\left(\Omega ; C\left([0, T] ; L^{2}(G)\right)\right) \bigcap L_{\mathbb{F}}^{2}\left(\Omega ; C^{1}\left([0, T] ; H^{-1}(G)\right)\right)
$$

and

$$
\mathcal{H}_{T}=L_{\mathbb{F}}^{2}\left(\Omega ; C^{1}\left([0, T] ; L^{2}(G)\right)\right) \bigcap L_{\mathbb{F}}^{2}\left(\Omega ; C\left([0, T] ; H_{0}^{1}(G)\right)\right) .
$$

Then $H_{T}$ and $\mathcal{H}_{T}$ are Banach spaces with the canonical norms. In this paper, we use the following notion of solution for equation (1.3).

Definition 1.1. A function $y \in H_{T}$ is called a solution to equation (1.3), if the following conditions hold:

(1) $y(0)=y_{0}$ and $y_{t}(0)=y_{1}$ in $G$, $\mathcal{P}$-a.s.

(2) For any $t \in(0, T)$ and $\varphi \in C^{2}(\bar{G}) \cap C_{0}^{1}(G)$, it holds that

$$
\begin{aligned}
& \left\langle y_{t}(t), \varphi\right\rangle_{H^{-1}(G), H_{0}^{1}(G)}-\left\langle y_{t}(0), \varphi\right\rangle_{H^{-1}(G), H_{0}^{1}(G)} \\
& =\int_{0}^{t} \int_{G}\left\{\sum_{i, j=1}^{n}\left[b^{i j}(x) \varphi_{x_{i}}(x)\right]_{x_{j}} y(s, x)+\left[b_{1}(s, x) y(s, x)+f(s, x)\right] \varphi(x)\right\} \mathrm{d} x \mathrm{~d} s \\
& +\int_{0}^{t} \int_{G}\left[b_{2}(s, x) y(s, x)+g(s, x)\right] \varphi(x) \mathrm{d} x \mathrm{~d} B(s), \quad \mathcal{P} \text {-a.s. }
\end{aligned}
$$

For any initial value $\left(y_{0}, y_{1}\right) \in L^{2}(G) \times H^{-1}(G)$, it is easy to show that equation (1.3) admits a unique solution $y \in H_{T}$.

Let $\Gamma_{0}$ be a part of the boundary of $G$ satisfying certain conditions, which will be specified later. For any given constant $\delta>0$, write

$$
\mathcal{O}_{\delta}\left(\Gamma_{0}\right)=\left\{x \in G ; \operatorname{dist}\left(x, \Gamma_{0}\right)<\delta\right\} .
$$

Put

$$
r_{1}=\left|b_{1}\right|_{L_{\mathbb{F}}^{\infty}\left(0, T ; L^{p}(G)\right)} \quad \text { and } \quad r_{2}=\left|b_{2}\right|_{L_{\mathbb{F}}^{\infty}\left(0, T ; L^{\infty}(G)\right)} .
$$

The main purpose of this paper is to establish the following inequality:

$$
\left|\left(y_{0}, y_{1}\right)\right|_{L^{2}(G) \times H^{-1}(G)} \leq C \mathrm{e}^{C\left(r_{1}^{\frac{1}{2-n / p}}+r_{2}^{2}\right)}\left[|y|_{L_{\mathbb{P}}^{2}\left(0, T ; L^{2}\left(\mathcal{O}_{\delta}\left(\Gamma_{0}\right)\right)\right)}+|(f, g)|_{\left(L_{\mathbb{P}}^{2}\left(0, T ; L^{2}(G)\right)\right)^{2}}\right],
$$


where $y$ is the solution to (1.3) corresponding to any given initial value $\left(y_{0}, y_{1}\right)$. Here and henceforth, $C$ denotes a generic positive constant (which may be different from line to line), depending only on $G, T, \Gamma_{0}, b^{i j}(i, j=$ $1, \ldots, n), \delta$, and $d(\cdot)$ and $\mu_{0}$ in Condition 1.2 (to be given later).

The inequality (1.7) is called an observability estimate for (1.3). For the case that $(f, g)=0$ in (1.3), this inequality means that the initial energy of a solution in the time $t=0$ can be bounded by its partial energy in the local observation domain $\mathcal{O}_{\delta}\left(\Gamma_{0}\right)$ in the time duration $[0, T]$. Such kind of inequalities are closely related to control and state observation problems of deterministic/stochastic hyperbolic equations. For example, they can be applied to a study of the controllability (e.g. $[1,2,4,9,15,17])$ and also inverse problems (e.g. $[7,8])$ for deterministic hyperbolic equations. There exist numerous works devoted to observability estimates for deterministic hyperbolic equations. However, there are only a very few works addressed to similar problems but for stochastic hyperbolic equations $[12,13,16]$.

Up to now, there are several methods to establish observability estimates for deterministic hyperbolic equations, such as the (Rellich-type) multiplier method [9], the non-harmonic Fourier series technique [14], the method of micro-local analysis [1] and the method of global Carleman estimate [15]. The multiplier method is only applicable to some very special hyperbolic equations. Indeed, even for a deterministic hyperbolic equation, the observability estimate cannot be derived by this method directly for the case that the coefficients of lower order terms depend on both the space variable and time variable. Also, the non-harmonic Fourier series technique has restrictions not only on the coefficients, but also on the spatial domain $G$ (requiring the domain $G$ to have some special shapes). Furthermore, since the propagation of singularities for stochastic partial differential equations is far from being well-understood, how to use the method of micro-local analysis in the stochastic framework to establish observability estimates is still unclear. Therefore, the Carleman estimate method turns out to be a useful tool to establish observability estimates for stochastic hyperbolic equations.

In [16], by means of a global Carleman estimate, a boundary observability estimate for equation (1.3) (with $\left(b^{i j}\right)_{1 \leq i, j \leq n}=I_{n}$, the $n \times n$ identity matrix) was obtained:

$$
\left|\left(y(T), y_{t}(T)\right)\right|_{L_{\mathcal{F}_{T}}^{2}\left(\Omega ; H_{0}^{1}(G) \times L^{2}(G)\right)} \leq C \mathrm{e}^{C\left(r_{1}^{2}+r_{2}^{2}\right)}\left(\left|\frac{\partial y}{\partial \nu}\right|_{L_{\mathbb{F}}^{2}\left(0, T ; L^{2}\left(\Gamma_{0}\right)\right)}+|(f, g)|_{\left(L_{\mathbb{F}}^{2}\left(0, T ; L^{2}(G)\right)\right)^{2}}\right),
$$

where $y$ solved equation (1.3) associated to an initial data $\left(y_{0}, y_{1}\right) \in H_{0}^{1}(G) \times L^{2}(G)$, and $\nu=\nu(x)=$ $\left(\nu^{1}, \nu^{2}, \ldots, \nu^{n}\right)$ denotes the unit outward normal vector of $G$ at $x \in \Gamma$. Also, in (1.8), $T$ was required to satisfy the condition:

$$
\frac{4+5 c}{9 c} \min _{x \in \bar{G}}\left|x-x_{0}\right|^{2}>c^{2} T^{2}>4 \max _{x \in \bar{G}}\left|x-x_{0}\right|^{2},
$$

for some $c \in(0,1)$ and $x_{0} \in \mathbb{R}^{n} \backslash \bar{G}$.

In [12], by virtue of another global Carleman estimate, the result in [16] was improved to the following boundary observability inequality:

$$
\left|\left(y_{0}, y_{1}\right)\right|_{H_{0}^{1}(G) \times L^{2}(G)} \leq C \mathrm{e}^{C\left(r_{1}^{\frac{1}{3 / 2-n / p}}+r_{2}^{2}\right)}\left(\left|\frac{\partial y}{\partial \nu}\right|_{L_{\mathbb{F}}^{2}\left(0, T ; L^{2}\left(\Gamma_{0}\right)\right)}+|(f, g)|_{\left(L_{\mathbb{F}}^{2}\left(0, T ; L^{2}(G)\right)\right)^{2}}\right),
$$

with $T>2 \max _{x \in \bar{G}}\left|x-x_{0}\right|$ (for the case that $\left(b^{i j}\right)_{1 \leq i, j \leq n}=I_{n}$ ). Notice that in (1.9), the power of $r_{1}$ is smaller than that in (1.8) (Indeed, $\frac{1}{3 / 2-n / p} \leq 2$ ). Also, an internal observability estimate was established in [12]:

$$
\left|\left(y_{0}, y_{1}\right)\right|_{H_{0}^{1}(G) \times L^{2}(G)} \leq C \mathrm{e}^{C\left(r_{1}^{\frac{1}{3 / 2-n / p}}+r_{2}^{2}\right)}\left(|\nabla y|_{L_{\mathbb{P}}^{2}\left(0, T ; L^{2}\left(\mathcal{O}_{\delta}\left(\Gamma_{0}\right)\right)\right)}+|(f, g)|_{\left(L_{\mathbb{F}}^{2}\left(0, T ; L^{2}(G)\right)\right)^{2}}\right) .
$$

The main difference between (1.7) and (1.10) is that the inequality (1.10) provides an observability estimate of the $H^{1}$-norm for solutions to equation (1.3), but the inequality (1.7) is an estimate of the $L^{2}$-norm type. Compared with the known inequality (1.10), the estimate (1.7) has more applications. For example, one application of (1.7) is the stabilization of stochastic hyperbolic equations (but the detailed analysis is beyond the 
scope of this paper and will be presented in our forthcoming work). On the other hand, the inequality (1.7) can be used to solve state observation problem (see [12] for example).

It is not clear whether it is possible to derive (1.7) from (1.10) directly. Indeed, this turns out to be very difficult, even for deterministic linear hyperbolic equations with some lower order terms (see $[4,6])$. Also, compared with the deterministic problem, the stochastic setting will bring this problem some new difficulties. Actually, as we shall see later, a suitable auxiliary optimal control problem (different from the deterministic context) has to be constructed to guarantee the adaptedness of the related stochastic processes.

Before giving our main result, let us first introduce some assumptions on $\left(b^{i j}\right)_{1 \leq i, j \leq n}(i, j=1, \ldots, n)$ and $T$.

Condition 1.2. There exists a positive function $d(\cdot) \in C^{2}(\bar{G})$ with the property that $\min _{x \in \bar{G}}|\nabla \mathrm{d}(x)|>0$ such that, for some constant $\mu_{0}>0$, the following compatibility condition is satisfied:

$$
\sum_{i, j=1}^{n} \sum_{i^{\prime}, j^{\prime}=1}^{n}\left[2 b^{i j^{\prime}}\left(b^{i^{\prime} j} d_{x_{i^{\prime}}}\right)_{x_{j^{\prime}}}-b_{x_{j^{\prime}}}^{i j} i^{i^{\prime} j^{\prime}} d_{x_{i^{\prime}}}\right] \xi^{i} \xi^{j} \geq \mu_{0} \sum_{i, j=1}^{n} b^{i j} \xi^{i} \xi^{j}, \quad \forall\left(x, \xi^{1}, \ldots, \xi^{n}\right) \in \bar{G} \times \mathbb{R}^{n} .
$$

In the sequel, we shall choose the set $\Gamma_{0}$ as follows:

$$
\Gamma_{0}=\left\{x \in \Gamma: \sum_{i, j=1}^{n} b^{i j}(x) d_{x_{i}}(x) \nu^{j}(x)>0\right\}
$$

where the function $d(\cdot)$ is given in Condition 1.2. Also, write

$$
G_{0}=\mathcal{O}_{\delta}\left(\Gamma_{0}\right) \quad \text { and } \quad \Sigma_{0}=\Gamma_{0} \times(0, T) .
$$

Remark 1.3. Notice that Condition 1.2 is a sufficient condition for establishing Carleman estimates for deterministic linear hyperbolic operators $\partial_{t}^{2}-\sum_{i, j=1}^{n} \partial_{x_{j}}\left(b^{i j} \partial_{x_{i}}\right)$. If $\left(b^{i j}\right)_{1 \leq i, j \leq n}=I_{n}$, then $\mathrm{d}(x)=\left|x-x_{0}\right|^{2}$ satisfies Condition 1.2 with $x_{0}$ being any given point in $\mathbb{R}^{n} \backslash \bar{G}$. On the other hand, Condition 1.2 can also be regarded as a special case of the pseudo-convexity condition in [5]. In fact, for the wave operator $\partial_{t}^{2}-\Delta$, if we set $a(x, \xi)=|\xi|^{2}$ and $\mathrm{d}(x)=\left|x-x_{0}\right|^{2}$, then it is easy to check that

$$
\{a,\{a, d\}\}(x, \xi)=4|\xi|^{2}>0, \quad \forall(x, \xi) \in \bar{G} \times\left(\mathbb{R}^{n} \backslash\{0\}\right),
$$

where $\{a, d\}$ denotes the Poisson bracket of $a$ and $d$, i.e.,

$$
\{a, d\}(x, \xi)=\sum_{j=1}^{n}\left(\frac{\partial a}{\partial \xi_{j}} \frac{\partial d}{\partial x_{j}}-\frac{\partial a}{\partial x_{j}} \frac{\partial d}{\partial \xi_{j}}\right), \quad \forall(x, \xi) \in \bar{G} \times \mathbb{R}^{n} \backslash\{0\} .
$$

Moreover, it is easy to see that there is no critical point of the function $d(\cdot)$ in $\bar{G}$.

Remark 1.4. In [4], Condition 1.2 was used to establish an internal observability estimate for deterministic hyperbolic equations. We refer to $[4,11]$ for more explanations on Condition 1.2 and some interesting nontrivial examples. Also, as ([11], Example 1.1) shows, there exists some example for which Condition 1.2 fails.

In what follows, set

$$
M_{0}=\min _{x \in \bar{G}} \sum_{i, j=1}^{n} b^{i j} d_{x_{i}} d_{x_{j}}, \quad M_{1}=\max _{x \in \bar{G}} \sum_{i, j=1}^{n} b^{i j} d_{x_{i}} d_{x_{j}} \quad \text { and } \quad d_{0}=\max _{x \in \Gamma} \sum_{i, j=1}^{n} b^{i j} d_{x_{i}} \nu^{j},
$$


and define ${ }^{3}$

$$
T_{0}=\max \left\{2 \sqrt{M_{1}}, 1+\frac{24 \sqrt{n} d_{0}}{\min \left\{1, s_{0}\right\}}\left(1+\frac{1}{s_{0}^{3 / 2}} \sum_{i, j=1}^{n}\left|b^{i j}\right|_{C(\bar{G})}+\frac{1}{s_{0}}\right)\right\},
$$

where $s_{0}$ is the constant appeared in (1.2).

Remark 1.5. It is easy to check that if $d(\cdot)$ satisfies Condition 1.2 , then for any $a \geq 1$ and $b \in \mathbb{R}$, the function $\tilde{d}(\cdot)=a d(\cdot)+b$ still satisfies this condition when $\mu_{0}$ is replaced by $a \mu_{0}$. Therefore, throughout this paper, we may assume that $d(\cdot)$ and $\mu_{0}$ satisfy that

$$
\mu_{0}>\frac{9 T_{0}^{2}}{M_{0}} \quad \text { and } \quad M_{0} \geq \max _{x \in \bar{G}} \mathrm{~d}(x) .
$$

The main result of this paper is stated as follows.

Theorem 1.6. Assume that Condition 1.2 holds. Then, for any $T>T_{0}$ (defined by (1.14)), the observability inequality (1.7) holds for any solution to equation (1.3).

Remark 1.7. The restriction on $\mathrm{T}$ in Theorem 1.6 is a technical condition, and $T_{0}$ is not sharp. However, this condition plays a key role in our Proof of Theorem 1.6. It is reasonable to expect that it can be improved to a better one as that in [12] (for the estimates (1.9) and (1.10)), but this is an unsolved problem.

Remark 1.8. The condition (1.15) is relevant to the interior behavior/property of the diffusion, and it will play a key role in the estimates on the energy terms (see (2.13)-(2.15) in the proof of Thm. 3.1). On the other hand, the assumption on the time $T$ in Theorem 1.6 is relevant to the diffusion/reflection on the boundary. This assumption will play a key role in the estimates on the boundary term (see Step 4 in the proof of Thm. 3.1). If one considers a special case, i.e. $\left(b^{i j}\right)_{1 \leq i, j \leq n}=I_{n}$, then $s_{0}=1$ and we take $\mathrm{d}(x)=\left|x-x_{0}\right|^{2}$, the corresponding condition on $T$ is the following:

$$
T>T_{0}=\max \left\{4 \max _{x \in \bar{G}}\left|x-x_{0}\right|, \quad 1+48 \sqrt{n}(n+2) \max _{x \in \Gamma}\left[\left(x-x_{0}\right) \cdot \nu(x)\right]\right\} .
$$

The rest of this paper is organized as follows. In Section 2, we present a key weighted identity for partial differential operators of second order with symmetric coefficients. Section 3 is devoted to establishing a Carleman estimate for deterministic hyperbolic equations in the $H^{1}$-space. In Section 4, an auxiliary optimal control problem is introduced and analyzed. In Section 5, a global Carleman estimate for stochastic hyperbolic equations in the $L^{2}$-space is derived. In Section 6, energy estimates for random hyperbolic equations and backward stochastic hyperbolic equations are given. Section 7 is devoted to a proof of our main result (i.e., Thm. 1.6). Finally, in Appendices A and B, we give the proofs of some technical results.

\section{A WEIGHTED IDENTITY FOR PARTIAL DIFFERENTIAL OPERATORS OF SECOND ORDER WITH SYMMETRIC COEFFICIENTS}

In this section, we show a pointwise weighted identity for partial differential operators of second order with symmetric coefficients, which will play a crucial role in the sequel.

\footnotetext{
${ }^{3}$ From the proof of ([4], Thm. 5.1), it is easy to see that the number $d_{0}$ defined in (1.13) is positive. Hence, the set $\Gamma_{0}$ given by (1.11) is not empty.
} 
Lemma 2.1. Assume that $a^{i j}=a^{j i} \in C^{2}\left(\mathbb{R}^{m}\right)(i, j=1,2, \ldots, m)$ for some $m \in \mathbb{N}, u, \ell \in C^{2}\left(\mathbb{R}^{m}\right)$ and $\Psi \in C^{1}\left(\mathbb{R}^{m}\right)$. Set $\theta=\mathrm{e}^{\ell}$ and $v=\theta u$. Then

$$
\begin{aligned}
& \theta^{2}\left|\sum_{i, j=1}^{m}\left(a^{i j} u_{x_{i}}\right)_{x_{j}}\right|^{2}+2 \sum_{j=1}^{m}\left[\sum_{i, i^{\prime}, j^{\prime}=1}^{m}\left(2 a^{i j} a^{i^{\prime} j^{\prime}} \ell_{x^{\prime}} v_{x_{i}} v_{x_{j^{\prime}}}-a^{i j} a^{i^{\prime} j^{\prime}} \ell_{x_{i}} v_{x_{i^{\prime}}} v_{x_{j^{\prime}}}\right)+\Psi \sum_{i=1}^{m} a^{i j} v_{x_{i}} v-\Lambda \sum_{i=1}^{m} a^{i j} \ell_{x_{i}} v^{2}\right]_{x_{j}} \\
& \geq 2 \sum_{i, j=1}^{m}\left[\sum_{i^{\prime}, j^{\prime}=1}^{m}\left(2 a^{i j^{\prime}}\left(a^{i^{\prime} j} \ell_{x_{i^{\prime}}}\right)_{x_{j^{\prime}}}-\left(a^{i j} a^{i^{\prime} j^{\prime}} \ell_{x_{i^{\prime}}}\right)_{x_{j^{\prime}}}\right)+\Psi a^{i j}\right] v_{x_{i}} v_{x_{j}}+2 \sum_{i, j=1}^{m} a^{i j} \Psi_{x_{j}} v v_{x_{i}}+B v^{2},
\end{aligned}
$$

where

$$
\Lambda=-\sum_{i, j=1}^{m}\left(a^{i j} \ell_{x_{i}} \ell_{x_{j}}-a_{x_{j}}^{i j} \ell_{x_{i}}-a^{i j} \ell_{x_{i} x_{j}}\right)-\Psi \quad \text { and } \quad B=2 \Lambda \Psi-2 \sum_{i, j=1}^{m}\left(\Lambda a^{i j} \ell_{x_{i}}\right)_{x_{j}} .
$$

Remark 2.2. Lemma 2.1 looks very similar to Theorem 4.1 in [4]. The only difference is about the regularity on the auxiliary function $\Psi$. In [4], $\Psi$ was required to be in $C^{2}\left(\mathbb{R}^{m}\right)$. But here we weaken this requirement to be $\Psi \in C^{1}\left(\mathbb{R}^{m}\right)$. Note that, the choice of $\Psi$ usually depends on coefficients of the principal operator under consideration (see Eq. (4.13) in [4]). Hence, this implies that we only need the $C^{2}$-regularity for coefficients of principal operators, rather than the $C^{3}$-regularity required in [4].

Proof of Lemma 2.1. Recalling $\theta=\mathrm{e}^{\ell}$ and $v=\theta u$, we see that $\theta u_{x_{i}}=v_{x_{i}}-\ell_{x_{i}} v(i=1,2, \ldots, m)$. Proceeding exactly as ([4], Thm. 4.1), we obtain that

$$
-\theta \sum_{i, j=1}^{m}\left(a^{i j} u_{x_{i}}\right)_{x_{j}}=I_{1}+I_{2}
$$

where

$$
I_{1}=-\sum_{i, j=1}^{m}\left(a^{i j} v_{x_{i}}\right)_{x_{j}}+\Lambda v \quad \text { and } \quad I_{2}=2 \sum_{i, j=1}^{m} a^{i j} \ell_{x_{i}} v_{x_{j}}+\Psi v
$$

This implies that

$$
\theta^{2}\left|\sum_{i, j=1}^{m}\left(a^{i j} u_{x_{i}}\right)_{x_{j}}\right|^{2}=\left|I_{1}\right|^{2}+2 I_{1} I_{2}+\left|I_{2}\right|^{2} \geq 2 I_{1} I_{2} .
$$

By virtue of ([4], Eq. (4.8)), a simple calculation shows that

$$
\begin{aligned}
I_{1} I_{2}= & 2 \sum_{i, j=1}^{m} a^{i j} \ell_{x_{i}} v_{x_{j}}\left(-\sum_{i, j=1}^{m}\left(a^{i j} v_{x_{i}}\right)_{x_{j}}+\Lambda v\right)+\Psi v\left(-\sum_{i, j=1}^{m}\left(a^{i j} v_{x_{i}}\right)_{x_{j}}+\Lambda v\right) \\
= & -\sum_{j=1}^{m}\left(2 \sum_{i, i^{\prime}, j^{\prime}=1}^{m} a^{i j} a^{i^{\prime} j^{\prime}} \ell_{x_{i^{\prime}}} v_{x_{i}} v_{x_{j^{\prime}}}-\sum_{i, i^{\prime}, j^{\prime}=1}^{m} a^{i j} a^{i^{\prime} j^{\prime}} \ell_{x_{i}} v_{x_{i^{\prime}}} v_{x_{j^{\prime}}}-\Lambda \sum_{i=1}^{m} a^{i j} \ell_{x_{i}} v^{2}\right)_{x_{j}} \\
& +\sum_{i, j, i^{\prime}, j^{\prime}=1}^{m}\left(2 a^{i j^{\prime}}\left(a^{i^{\prime} j} \ell_{x_{i^{\prime}}}\right)_{x_{j^{\prime}}}-\left(a^{i j} a^{i^{\prime} j^{\prime}} \ell_{x_{i^{\prime}}}\right)_{x_{j^{\prime}}}\right) v_{x_{i}} v_{x_{j}}-\sum_{i, j=1}^{m}\left(\Lambda a^{i j} \ell_{x_{i}}\right)_{x_{j}} v^{2} \\
& -\sum_{i, j=1}^{m}\left(\Psi a^{i j} v v_{x_{i}}\right)_{x_{j}}+\Psi \sum_{i, j=1}^{m} a^{i j} v_{x_{i}} v_{x_{j}}+\sum_{i, j=1}^{m} a^{i j} \Psi_{x_{j}} v v_{x_{i}}+\Lambda \Psi v^{2} .
\end{aligned}
$$

Combining (2.5) with (2.4), we obtain the desired inequality (2.1). 


\section{A Carleman estimate for Deterministic hyperbolic equations IN THE $H^{1}$-NORM}

This section is addressed to deriving a Carleman estimate for the following (deterministic) hyperbolic equation:

$$
\begin{cases}u_{t t}-\sum_{i, j=1}^{n}\left(b^{i j} u_{x_{i}}\right)_{x_{j}}=F & \text { in } Q, \\ u=0 & \text { on } \Sigma, \\ u(0)=u_{0}, u_{t}(0)=u_{1} & \text { in } G,\end{cases}
$$

where $\left(u_{0}, u_{1}\right) \in H_{0}^{1}(G) \times L^{2}(G), F \in L^{2}(Q)$, and $b^{i j}(i, j=1, \ldots, n)$ satisfy (1.1), (1.2) and the Condition 1.2.

As in Theorem 1.6, we assume that $T>T_{0}$ (defined in (1.14)), and $G_{0}, \mu_{0}$ and $d(\cdot)$ are given in (1.12) and Condition 1.2. By (1.15), it is easy to see that $\frac{T_{0}}{T}<\frac{\sqrt{\mu_{0} M_{0}}}{3 T}$. Hence, we can choose a constant $c_{1} \in$ $\left(T_{0} / T, \min \left\{1, \frac{\sqrt{\mu_{0} M_{0}}}{3 T}\right\}\right)$. Now, for any given constant $c_{0} \in(0,1)$ and parameter $\lambda>0$, we choose the weight function $\theta$ and the auxiliary function $\Psi$ (appeared in Lem. 2.1) as follows:

$$
\left\{\begin{array}{l}
\theta(t, x)=\mathrm{e}^{\ell(t, x)} \\
\ell(t, x)=\lambda \phi(t, x) \\
\phi(t, x)=\mathrm{d}(x)-c_{1}(t-T / 2)^{2} \\
\Psi(x)=\lambda\left(\sum_{i, j=1}^{n}\left(b^{i j} d_{x_{i}}\right)_{x_{j}}-2 c_{1}-c_{0}\right) .
\end{array}\right.
$$

We have the following global Carleman estimate for equation (3.1).

Theorem 3.1. Assume that Condition 1.2 holds. Then, there is a positive constant $\lambda_{0}$, such that for any $T>T_{0}$ and $\lambda \geq \lambda_{0}$, any solution $u$ to (3.1) satisfies that

$$
\int_{Q} \mathrm{e}^{2 \lambda \phi}\left[\lambda\left(u_{t}^{2}+|\nabla u|^{2}\right)+\lambda^{3} u^{2}\right] \mathrm{d} x \mathrm{~d} t \leq C\left[\int_{Q} \mathrm{e}^{2 \lambda \phi} F^{2} \mathrm{~d} x \mathrm{~d} t+\lambda^{2} \int_{0}^{T} \int_{G_{0}} \mathrm{e}^{2 \lambda \phi}\left(u_{t}^{2}+\lambda^{2} u^{2}\right) \mathrm{d} x \mathrm{~d} t\right] .
$$

Remark 3.2. Notice that Theorem 3.1 is an improvement of ([4], Thm. 5.1). Indeed, in ([4], Thm. 5.1), the global Carleman estimate (3.3) for a deterministic hyperbolic equation was established under the additional condition that $u(0)=u(T)=0$ in $G$. However, this condition seems too strong to be satisfied in applications (see, for example Eq. (7.5) in [4]). Therefore, it is necessary to establish the global Carleman estimate (3.3) without this restriction.

In the rest of this section, we give a Proof of Theorem 3.1.

Proof of Theorem 3.1. The proof is long and therefore we divide it into four steps.

Step 1. A pointwise inequality for hyperbolic operators. In Lemma 2.1, we choose $m=n+1$ and $\left(a^{i j}\right)_{m \times m}=\left(\begin{array}{cc}-1 & 0 \\ 0 & \left(b^{i j}\right)_{n \times n}\end{array}\right)$, and $\theta, \ell, \phi$ and $\Psi \equiv \Psi(x)$ being given as in (3.2). Then, by a simple calculation, we have the following weighted inequality for the hyperbolic operator, which is very similar to ([4], Cor. 4.2), 
except some different lower order terms.

$$
\begin{aligned}
& \mathrm{e}^{2 \lambda \phi}\left|u_{t t}-\sum_{i, j=1}^{n}\left(b^{i j} u_{x_{i}}\right)_{x_{j}}\right|^{2}+2 \sum_{j=1}^{n}\left(2 \sum_{i, i^{\prime}, j^{\prime}=1}^{n} b^{i j} b^{i^{\prime} j^{\prime}} \ell_{x_{i^{\prime}}} v_{x_{i}} v_{x_{j^{\prime}}}-\sum_{i=1}^{n} b^{i j} \Lambda \ell_{x_{i}} v^{2}\right. \\
& \left.-\sum_{i, i^{\prime}, j^{\prime}=1}^{n} b^{i j} b^{i^{\prime} j^{\prime}} \ell_{x_{i}} v_{x_{i^{\prime}}} v_{x_{j^{\prime}}}+\Psi v \sum_{i=1}^{n} b^{i j} v_{x_{i}}-2 \ell_{t} v_{t} \sum_{i=1}^{n} b^{i j} v_{x_{i}}+\sum_{i=1}^{n} b^{i j} \ell_{x_{i}} v_{t}^{2}\right)_{x_{j}}+2 M_{t} \\
& \geq 2\left[\ell_{t t}+\sum_{i, j=1}^{n}\left(b^{i j} \ell_{x_{i}}\right)_{x_{j}}-\Psi\right] v_{t}^{2}-8 \sum_{i, j=1}^{n} b^{i j} \ell_{x_{j}} v_{x_{i}} v_{t}+2 \sum_{i, j=1}^{n} b^{i j} \Psi_{x_{j}} v v_{x_{i}}+B v^{2} \\
& \quad+2 \sum_{i, j=1}^{n}\left\{b^{i j} \ell_{t t}+\sum_{i^{\prime}, j^{\prime}=1}^{n}\left[2 b^{i j^{\prime}}\left(b^{i^{\prime} j} \ell_{x_{i^{\prime}}}\right)_{x_{j^{\prime}}}-\left(b^{i j} b^{i^{\prime} j^{\prime}} \ell_{x_{i^{\prime}}}\right)_{x_{j^{\prime}}}\right]+\Psi b^{i j}\right\} v_{x_{i}} v_{x_{j}},
\end{aligned}
$$

where

$$
\left\{\begin{array}{l}
\Lambda=\ell_{t}^{2}-\ell_{t t}-\sum_{i, j=1}^{n}\left(b^{i j} \ell_{x_{i}} \ell_{x_{j}}-b_{x_{j}}^{i j} \ell_{x_{i}}-b^{i j} \ell_{x_{i} x_{j}}\right)-\Psi \\
M=\ell_{t}\left(v_{t}^{2}+\sum_{i, j=1}^{n} b^{i j} v_{x_{i}} v_{x_{j}}\right)-2 \sum_{i, j=1}^{n} b^{i j} \ell_{x_{i}} v_{x_{j}} v_{t}-\Psi v v_{t}+\Lambda \ell_{t} v^{2} \\
B=2\left[\Lambda \Psi+\left(\Lambda \ell_{t}\right)_{t}-\sum_{i, j=1}^{n}\left(\Lambda b^{i j} \ell_{x_{i}}\right)_{x_{j}}\right] .
\end{array}\right.
$$

Step 2. Estimates on "the energy terms". First, by the definitions of $\Psi$ and $\ell$, it is easy to show that

$$
2\left[\ell_{t t}+\sum_{i, j=1}^{n}\left(b^{i j} \ell_{x_{i}}\right)_{x_{j}}-\Psi\right]=-4 \lambda c_{1}+2 \sum_{i, j=1}^{n}\left(\lambda b^{i j} d_{x_{i}}\right)_{x_{j}}-2 \Psi=2 \lambda c_{0} .
$$

Further,

$$
\begin{aligned}
& 2 \sum_{i, j=1}^{n}\left\{b^{i j} \ell_{t t}+\sum_{i^{\prime}, j^{\prime}=1}^{n}\left[2 b^{i j^{\prime}}\left(b^{i^{\prime} j} \ell_{x_{i^{\prime}}}\right)_{x_{j^{\prime}}}-\left(b^{i j} b^{i^{\prime} j^{\prime}} \ell_{x_{i^{\prime}}}\right)_{x_{j^{\prime}}}\right]+\Psi b^{i j}\right\} v_{x_{i}} v_{x_{j}} \\
& =2 \sum_{i, j=1}^{n}\left[-2 \lambda c_{1} b^{i j}+\sum_{i^{\prime}, j^{\prime}=1}^{n} \lambda b^{i j}\left(b^{i^{\prime} j^{\prime}} d_{x_{i^{\prime}}}\right)_{x_{j^{\prime}}}-2 \lambda c_{1} b^{i j}-c_{0} \lambda b^{i j}\right. \\
& \left.\quad+2 \lambda \sum_{i^{\prime}, j^{\prime}=1}^{n} b^{i j^{\prime}}\left(b^{i^{\prime} j} d_{x_{i^{\prime}}}\right)_{x_{j^{\prime}}}-\lambda \sum_{i^{\prime}, j^{\prime}=1}^{n}\left(b^{i j} b^{i^{\prime} j^{\prime}} d_{x_{i^{\prime}}}\right)_{x_{j^{\prime}}}\right] v_{x_{i}} v_{x_{j}} \\
& \geq 2 \lambda \mu_{0} \sum_{i, j=1}^{n} b^{i j} v_{x_{i}} v_{x_{j}}-\left(8 c_{1}+2 c_{0}\right) \lambda \sum_{i, j=1}^{n} b^{i j} v_{x_{i}} v_{x_{j}}=2 \lambda\left(\mu_{0}-4 c_{1}-c_{0}\right) \sum_{i, j=1}^{n} b^{i j} v_{x_{i}} v_{x_{j}} .
\end{aligned}
$$

Further, by (3.2) and (3.5), we obtain that

$$
\Lambda=\ell_{t}^{2}-\ell_{t t}-\sum_{i, j=1}^{n}\left(b^{i j} \ell_{x_{i}} \ell_{x_{j}}-b_{x_{j}}^{i j} \ell_{x_{i}}-b^{i j} \ell_{x_{i} x_{j}}\right)-\Psi=\lambda^{2}\left[c_{1}^{2}(2 t-T)^{2}-\sum_{i, j=1}^{n} b^{i j} d_{x_{i}} d_{x_{j}}\right]+O(\lambda) .
$$

Hence,

$$
B=2 \lambda^{3}\left[\left(4 c_{1}+c_{0}\right) \sum_{i, j=1}^{n} b^{i j} d_{x_{i}} d_{x_{j}}+\sum_{i, j=1}^{n} b^{i j} d_{x_{i}}\left(\sum_{i^{\prime}, j^{\prime}=1}^{n} b^{i^{\prime} j^{\prime}} d_{x_{i^{\prime}}} d_{x_{j^{\prime}}}\right)_{x_{j}}-\left(8 c_{1}+c_{0}\right) c_{1}^{2}(2 t-T)^{2}\right]+O\left(\lambda^{2}\right) .
$$


Proceeding the same analysis as (11.6)-(11.8) in [4], we have that

$$
\sum_{i, j=1}^{n} \sum_{i^{\prime}, j^{\prime}=1}^{n} b^{i j} d_{x_{i}}\left(b^{i^{\prime} j^{\prime}} d_{x_{i^{\prime}}} d_{x_{j^{\prime}}}\right)_{x_{j}} \geq \mu_{0} \sum_{i, j=1}^{n} b^{i j} d_{x_{i}} d_{x_{j}}
$$

By (3.8), (3.9) and (1.15), noticing that $c_{1}<\frac{\sqrt{\mu_{0} M_{0}}}{3 T}$, we find that for any $T>T_{0}$,

$$
B \geq 2 \lambda^{3}\left(4 c_{1}+c_{0}\right) \sum_{i, j=1}^{n} b^{i j} d_{x_{i}} d_{x_{j}}+O\left(\lambda^{2}\right) .
$$

On the other hand, by (1.15), it is easy to see that

$$
\frac{\mu_{0} M_{0}}{8 c_{1}+c_{0}}>\frac{\mu_{0} M_{0}}{9}>T_{0}^{2} \geq 4 M_{1} \geq 4 M_{0}
$$

which implies that

$$
\mu_{0}-4 c_{1}-c_{0}>\mu_{0}-32 c_{1}-4 c_{0}>0 .
$$

Therefore, combining (3.6), (3.7) (3.10) and (3.11) with (3.4), we conclude that for any $T>T_{0}$, there is a $\lambda_{1}>0$ and $c^{*}>0$, such that for any $\lambda \geq \lambda_{1}$,

$$
\begin{aligned}
& 2\left[\ell_{t t}+\sum_{i, j=1}^{n}\left(b^{i j} \ell_{x_{i}}\right)_{x_{j}}-\Psi\right] v_{t}^{2}-8 \sum_{i, j=1}^{n} b^{i j} \ell_{x_{j} t} v_{x_{i}} v_{t}+2 \sum_{i, j=1}^{n} b^{i j} \Psi_{x_{j}} v v_{x_{i}}+B v^{2} \\
& \quad+2 \sum_{i, j=1}^{n}\left\{b^{i j} \ell_{t t}+\sum_{i^{\prime}, j^{\prime}=1}^{n}\left[2 b^{i j^{\prime}}\left(b^{i^{\prime} j} \ell_{x_{i^{\prime}}}\right)_{x_{j^{\prime}}}-\left(b^{i j} b^{i^{\prime} j^{\prime}} \ell_{x_{i^{\prime}}}\right)_{x_{j^{\prime}}}\right]+\Psi b^{i j}\right\} v_{x_{i}} v_{x_{j}} \\
& \geq c^{*} \lambda\left(v_{t}^{2}+|\nabla v|^{2}+\lambda^{2} v^{2}\right) .
\end{aligned}
$$

Integrating (3.12) in $Q$ and noting that $v=\mathrm{e}^{\lambda \phi} u$ on $\Sigma$, by (3.1) and (3.4), we obtain that

$$
c^{*} \lambda \int_{Q}\left(v_{t}^{2}+|\nabla v|^{2}+\lambda^{2} v^{2}\right) \mathrm{d} x \mathrm{~d} t \leq \int_{Q} \mathrm{e}^{2 \lambda \phi}|F|^{2} \mathrm{~d} x \mathrm{~d} t+2 \int_{Q} M_{t} \mathrm{~d} x \mathrm{~d} t+2 \lambda d_{0} \int_{\Sigma_{0}} \sum_{i, j=1}^{n} b^{i j} \nu^{i} \nu^{j} \mathrm{e}^{2 \lambda \phi}\left|\frac{\partial u}{\partial \nu}\right|^{2} \mathrm{~d} \Sigma .
$$

Here we use the following identity:

$$
\int_{\Sigma_{i, j, i^{\prime}, j^{\prime}=1}} \sum^{n}\left(2 b^{i j} b^{i^{\prime} j^{\prime}} \ell_{x_{i^{\prime}}} v_{x_{i}} v_{x_{j^{\prime}}}-b^{i j} b^{i^{\prime} j^{\prime}} \ell_{x_{i}} v_{x_{i^{\prime}}} v_{x_{j^{\prime}}}\right) \cdot \nu^{j} \mathrm{~d} \Sigma=\lambda \int_{\Sigma} \sum_{i, j=1}^{n} b^{i j} \nu^{i} \nu^{j} \sum_{i^{\prime}, j^{\prime}=1}^{n} b^{i^{\prime} j^{\prime}} d_{x_{i^{\prime}}} j^{j^{\prime}}\left|\frac{\partial v}{\partial \nu}\right|^{2} \mathrm{~d} \Sigma .
$$

Step 3. Estimates on "the spatial boundary term". Let us estimate the last term of (3.13). Similar to the proof of (11.15) in [4], we choose functions $h_{0} \in C^{1}\left(\bar{G} ;[0,1]^{n}\right)$ and $\rho \in C^{2}(\bar{G} ;[0,1])$, such that $h_{0}=\nu$ on $\Gamma$, and for the same $\delta$ appeared in (1.12),

$$
\begin{cases}\rho(x) \equiv 1, & x \in \mathcal{O}_{\delta / 3}\left(\Gamma_{0}\right) \cap G, \\ \rho(x) \equiv 0, & x \in G \backslash \mathcal{O}_{\delta / 2}\left(\Gamma_{0}\right) .\end{cases}
$$

Let $h=h_{0} \rho \mathrm{e}^{2 \lambda \phi}$. Then, by ([4], Lem. 3.2) (with $g$ and $a^{i j}$ replaced by $h$ and $b^{i j}$, respectively),

$$
\begin{aligned}
\int_{\Sigma} \sum_{i, j=1}^{n} b^{i j} \nu^{i} \nu^{j} \rho \mathrm{e}^{2 \lambda \phi}\left|\frac{\partial u}{\partial \nu}\right|^{2} \mathrm{~d} x \mathrm{~d} t=- & \int_{Q}\left\{2\left[F h \cdot \nabla u-\left(u_{t} h \cdot \nabla u\right)_{t}+u_{t} h_{t} \cdot \nabla u-(\nabla \cdot h) u_{t}^{2}\right]\right. \\
& \left.-\sum_{i, j, k=1}^{n} b^{i j} u_{x_{i}} u_{x_{k}} \frac{\partial h^{k}}{\partial x_{j}}+\sum_{i, j=1}^{n} u_{x_{i}} u_{x_{j}} \nabla \cdot\left(b^{i j} h\right)\right\} \mathrm{d} x \mathrm{~d} t .
\end{aligned}
$$


Therefore, it is easy to check that

$$
\begin{aligned}
& \int_{\Sigma} \sum_{i, j=1}^{n} b^{i j} \nu^{i} \nu^{j} \rho \mathrm{e}^{2 \lambda \phi}\left|\frac{\partial u}{\partial \nu}\right|^{2} \mathrm{~d} x \mathrm{~d} t \\
& \leq \frac{C}{\lambda} \int_{Q} \mathrm{e}^{2 \lambda \phi} F^{2} \mathrm{~d} x \mathrm{~d} t+\lambda \int_{0}^{T} \int_{\mathcal{O}_{\delta / 2}\left(\Gamma_{0}\right) \cap G} \mathrm{e}^{2 \lambda \phi}|\nabla u|^{2} \mathrm{~d} x \mathrm{~d} t+\left.2 \int_{G} u_{t} h \cdot \nabla u \mathrm{~d} x\right|_{0} ^{T} \\
& \quad+4 \sqrt{n} \lambda \int_{0}^{T} \int_{\mathcal{O}_{\delta / 2}\left(\Gamma_{0}\right) \cap G} \mathrm{e}^{2 \lambda \phi} c_{1} T\left|u_{t}\right||\nabla u| \mathrm{d} x \mathrm{~d} t+C \lambda \int_{0}^{T} \int_{\mathcal{O}_{\delta / 2}\left(\Gamma_{0}\right) \cap G} \mathrm{e}^{2 \lambda \phi} u_{t}^{2} \mathrm{~d} x \mathrm{~d} t \\
& +\left(6 \sqrt{n} \lambda|\nabla d| \sum_{i, j=1}^{n}\left|b^{i j}\right|_{C(\bar{G})}+C\right) \int_{0}^{T} \int_{\mathcal{O}_{\delta / 2}\left(\Gamma_{0}\right) \cap G} \mathrm{e}^{2 \lambda \phi}|\nabla u|^{2} \mathrm{~d} x \mathrm{~d} t \\
& \leq C \\
& \quad\left(\frac{1}{\lambda} \int_{Q} \mathrm{e}^{2 \lambda \phi} F^{2} \mathrm{~d} x \mathrm{~d} t+\lambda \int_{0}^{T} \int_{\mathcal{O}_{\delta / 2}\left(\Gamma_{0}\right) \cap G} \mathrm{e}^{2 \lambda \phi} u_{t}^{2} \mathrm{~d} x \mathrm{~d} t\right)+\left.2 \int_{G} u_{t} h \cdot \nabla u \mathrm{~d} x\right|_{0} ^{T} \\
& +6 \sqrt{n} \lambda\left(|\nabla d| \sum_{i, j=1}^{n}\left|b^{i j}\right|_{C(\bar{G})}+1\right) \int_{0}^{T} \int_{\mathcal{O}_{\delta / 2}\left(\Gamma_{0}\right) \cap G} \mathrm{e}^{2 \lambda \phi}|\nabla u|^{2} \mathrm{~d} x \mathrm{~d} t .
\end{aligned}
$$

By (3.13) and the above inequality, we get that

$$
\begin{aligned}
& c^{*} \lambda \int_{Q}\left(v_{t}^{2}+|\nabla v|^{2}+\lambda^{2} v^{2}\right) \mathrm{d} x \mathrm{~d} t \\
& \leq C\left(\int_{Q} \mathrm{e}^{2 \lambda \phi} F^{2} \mathrm{~d} x \mathrm{~d} t+\lambda^{2} \int_{0}^{T} \int_{G_{0}} \mathrm{e}^{2 \lambda \phi} u_{t}^{2} \mathrm{~d} x \mathrm{~d} t\right)+\left.4 \lambda d_{0} \int_{G} u_{t} h \cdot \nabla u \mathrm{~d} x\right|_{0} ^{T}+\left.2 \int_{G} \tilde{M} \mathrm{~d} x\right|_{0} ^{T} \\
& \quad+12 \sqrt{n} \lambda^{2} d_{0}\left(|\nabla d| \sum_{i, j=1}^{n}\left|b^{i j}\right|_{C(\bar{G})}+1\right) \int_{0}^{T} \int_{\mathcal{O}_{\delta / 2}\left(\Gamma_{0}\right) \cap G} \mathrm{e}^{2 \lambda \phi}|\nabla u|^{2} \mathrm{~d} x \mathrm{~d} t
\end{aligned}
$$

where $\tilde{M}=M+2 \lambda d_{0} u_{t} h \cdot \nabla u$.

Next, let us estimate the last term in (3.14). Put $\eta(t, x)=\rho_{1}^{2} \mathrm{e}^{2 \lambda \phi}$, where $\rho_{1} \in C^{2}(\bar{G} ;[0,1])$ satisfies that

$$
\left\{\begin{array}{l}
\rho_{1}(x) \equiv 1, \quad x \in \mathcal{O}_{\delta / 2}\left(\Gamma_{0}\right) \cap G \\
\rho_{1}(x) \equiv 0, \quad x \in G \backslash G_{0}
\end{array}\right.
$$

By (3.1), we have that

$$
\begin{aligned}
& \int_{Q} \eta u F \mathrm{~d} x \mathrm{~d} t=\int_{Q} \eta u\left(u_{t t}-\sum_{i, j=1}^{n}\left(b^{i j} u_{x_{i}}\right)_{x_{j}}\right) \mathrm{d} x \mathrm{~d} t \\
& =\int_{Q}\left(\eta u u_{t}\right)_{t} \mathrm{~d} x \mathrm{~d} t-\int_{Q} u_{t}\left(\eta_{t} u+\eta u_{t}\right) \mathrm{d} x \mathrm{~d} t+\int_{Q} \eta \sum_{i, j=1}^{n} b^{i j} u_{x_{i}} u_{x_{j}} \mathrm{~d} x \mathrm{~d} t+\int_{Q} u \sum_{i, j=1}^{n} b^{i j} u_{x_{i}} \eta_{x_{j}} \mathrm{~d} x \mathrm{~d} t .
\end{aligned}
$$

This implies that

$$
\int_{0}^{T} \int_{\mathcal{O}_{\delta / 2}\left(\Gamma_{0}\right) \cap G} \mathrm{e}^{2 \lambda \phi}|\nabla u|^{2} \mathrm{~d} x \mathrm{~d} t \leq C\left[\frac{1}{\lambda^{2}} \int_{Q} \mathrm{e}^{2 \lambda \phi}|F|^{2} \mathrm{~d} x \mathrm{~d} t+\int_{0}^{T} \int_{G_{0}} \mathrm{e}^{2 \lambda \phi}\left(\lambda^{2} u^{2}+u_{t}^{2}\right) \mathrm{d} x \mathrm{~d} t\right]-\frac{1}{s_{0}} \int_{Q}\left(\eta u u_{t}\right)_{t} \mathrm{~d} x \mathrm{~d} t .
$$


Combining (3.14) with (3.15), we end up with

$$
c^{*} \lambda \int_{Q}\left(v_{t}^{2}+|\nabla v|^{2}+\lambda^{2} v^{2}\right) \mathrm{d} x \mathrm{~d} t \leq C \int_{Q} \mathrm{e}^{2 \lambda \phi}|F|^{2} \mathrm{~d} x \mathrm{~d} t+\left.\int_{G} \bar{M} \mathrm{~d} x\right|_{0} ^{T}+C \lambda^{2} \int_{0}^{T} \int_{G_{0}} \mathrm{e}^{2 \lambda \phi}\left(u_{t}^{2}+\lambda^{2} u^{2}\right) \mathrm{d} x \mathrm{~d} t,
$$

where

$$
\bar{M}=M+2 d_{0} \lambda u_{t} h \cdot \nabla u-\frac{1}{s_{0}}\left[12 \sqrt{n} \lambda^{2} d_{0}\left(|\nabla d| \sum_{i, j=1}^{n}\left|b^{i j}\right|_{C(\bar{G})}+1\right)\right] \eta u u_{t} .
$$

Step 4. Estimates on "the time boundary term". Let us estimate $\bar{M}(0, x)$ and $\bar{M}(T, x)$, respectively. By (1.15) and the definition of $M$ in (3.5), we have that

$$
\begin{aligned}
M(0, x) \geq & {\left.\left[\lambda c_{1} T\left(v_{t}^{2}+\sum_{i, j=1}^{n} b^{i j} v_{x_{i}} v_{x_{j}}\right)-2 \lambda \sum_{i, j=1}^{n} b^{i j} d_{x_{i}} v_{x_{j}} v_{t}\right]\right|_{t=0} } \\
& +\left.\left[O\left(\lambda^{2}\right) v^{2}-v_{t}^{2}+c_{1} T \lambda^{3}\left(c_{1}^{2} T^{2}-\sum_{i, j=1}^{n} b^{i j} d_{x_{i}} d_{x_{j}}\right) v^{2}\right]\right|_{t=0} \\
\geq & \left.\lambda\left[c_{1} T-\left(\sum_{i, j=1}^{n} b^{i j} d_{x_{i}} d_{x_{j}}\right)^{\frac{1}{2}}\right]\left(v_{t}^{2}+\sum_{i, j=1}^{n} b^{i j} v_{x_{i}} v_{x_{j}}\right)\right|_{t=0} \\
& +\left.\left[O\left(\lambda^{2}\right) v^{2}-v_{t}^{2}+c_{1} T \lambda^{3}\left(c_{1}^{2} T^{2}-\sum_{i, j=1}^{n} b^{i j} d_{x_{i}} d_{x_{j}}\right) v^{2}\right]\right|_{t=0} .
\end{aligned}
$$

Noting that by (1.14) and $c_{1}>T_{0} / T$, we have

$$
c_{1} T>2 \sqrt{M_{1}} \geq 2\left(\sum_{i, j=1}^{n} b^{i j} d_{x_{i}} d_{x_{j}}\right)^{\frac{1}{2}} \geq 2 \sqrt{s_{0}}|\nabla d| .
$$

This implies that

$$
M(0, x) \geq\left.\left[\frac{1}{2} \lambda c_{1} T \min \left\{1, s_{0}\right\}\left(v_{t}^{2}+|\nabla v|^{2}\right)+\frac{3}{4} \lambda^{3} c_{1}^{3} T^{3} v^{2}+O\left(\lambda^{2}\right) v^{2}-v_{t}^{2}\right]\right|_{t=0} .
$$

Further,

$$
\begin{aligned}
\left.2 d_{0} \lambda u_{t} h \cdot \nabla u\right|_{t=0} & \geq-\left.\sqrt{n} d_{0} \lambda \mathrm{e}^{2 \lambda \phi}\left(u_{t}^{2}+|\nabla u|^{2}\right)\right|_{t=0} \\
& \geq-\left.2 \sqrt{n} d_{0} \lambda\left(v_{t}^{2}+\lambda^{2} c_{1}^{2} T^{2} v^{2}+|\nabla v|^{2}+\lambda^{2}|\nabla d|^{2} v^{2}\right)\right|_{t=0}
\end{aligned}
$$

On the other hand,

$$
\begin{aligned}
& -\frac{1}{s_{0}}\left[12 \sqrt{n} \lambda^{2} d_{0}\left(|\nabla d| \sum_{i, j=1}^{n}\left|b^{i j}\right|_{C(\bar{G})}+1\right)\right] \eta u u_{t} \\
& =-\left.\frac{1}{s_{0}}\left[12 \sqrt{n} \lambda^{2} d_{0}\left(|\nabla d| \sum_{i, j=1}^{n}\left|b^{i j}\right|_{C(\bar{G})}+1\right)\right] \rho_{1}^{2}\left(v v_{t}-\lambda c_{1} T v^{2}-\frac{v_{t}^{2}}{\lambda c_{1} T}+\frac{v_{t}^{2}}{\lambda c_{1} T}\right)\right|_{t=0} \\
& \geq-\left.\frac{1}{s_{0} c_{1} T} \lambda\left[12 \sqrt{n} d_{0}\left(|\nabla d| \sum_{i, j=1}^{n}\left|b^{i j}\right|_{C(\bar{G})}+1\right)\right] v_{t}^{2}\right|_{t=0} .
\end{aligned}
$$


Therefore, by (3.18)-(3.20), we get that

$$
\bar{M}(0, x) \geq\left.\left(\lambda F_{1} v_{t}^{2}+\lambda F_{2}|\nabla v|^{2}+\lambda^{3} F_{3} v^{2}+O\left(\lambda^{2}\right) v^{2}+O(1) v_{t}^{2}\right)\right|_{t=0},
$$

where

$$
\left\{\begin{array}{l}
F_{1}=\frac{1}{2} c_{1} T \min \left\{1, s_{0}\right\}-2 \sqrt{n} d_{0}-\frac{1}{s_{0} c_{1} T}\left[12 \sqrt{n} d_{0}\left(|\nabla d| \sum_{i, j=1}^{n}\left|b^{i j}\right|_{C(\bar{G})}+1\right)\right], \\
F_{2}=\frac{1}{2} c_{1} T \min \left\{1, s_{0}\right\}-2 \sqrt{n} d_{0}, \quad F_{3}=\frac{3}{4} c_{1}^{3} T^{3}-2 \sqrt{n} d_{0}\left(c_{1}^{2} T^{2}+|\nabla d|^{2}\right) .
\end{array}\right.
$$

By (3.17) and (1.14), for any $T>T_{0}$, it holds that $c_{1} T>1$,

$$
F_{1} \geq \frac{1}{2} c_{1} T \min \left\{1, s_{0}\right\}-2 \sqrt{n} d_{0}-\frac{6 \sqrt{n} d_{0}}{s_{0}^{3 / 2}} \sum_{i, j=1}^{n}\left|b^{i j}\right|_{C(\bar{G})}-\frac{12 \sqrt{n} d_{0}}{s_{0}}>0,
$$

and therefore, $F_{2}>0$. Moreover,

$$
F_{3} \geq \frac{3}{4} c_{1}^{3} T^{3}-2 \sqrt{n} d_{0} c_{1}^{2} T^{2}\left(1+\frac{1}{4 s_{0}}\right)=\frac{3}{4} c_{1}^{2} T^{2}\left[c_{1} T-\frac{8}{3} \sqrt{n} d_{0}\left(1+\frac{1}{4 s_{0}}\right)\right]>0,
$$

where we use the following fact:

$$
c_{1} T>\frac{4 \sqrt{n}}{\min \left\{1, s_{0}\right\}} d_{0}=\frac{8 \sqrt{n}}{3 \min \left\{1, s_{0}\right\}} d_{0}+\frac{4 \sqrt{n}}{3 \min \left\{1, s_{0}\right\}} d_{0} \geq \frac{8 \sqrt{n}}{3} d_{0}+\frac{4 \sqrt{n}}{3 s_{0}} d_{0}=\frac{8}{3} \sqrt{n} d_{0}\left(1+\frac{1}{2 s_{0}}\right) .
$$

Finally, by (1.14), one can find constants $C>0$ and $\lambda_{2}>0$ such that for any $\lambda \geq \lambda_{2}$,

$$
\bar{M}(0, \cdot) \geq 0 .
$$

Meanwhile, noting that $\ell(T, x)=-\ell(0, x)$, we have that there is a constant $\lambda_{3}>0$ such that for any $\lambda \geq \lambda_{3}$,

$$
\bar{M}(T, \cdot) \leq 0
$$

Combining (3.23) and (3.24) with (3.16), and noting that $v=\mathrm{e}^{\lambda \phi} u$, for any $\lambda \geq \lambda_{0}=\max \left\{\lambda_{1}, \lambda_{2}, \lambda_{3}\right\}$, we end up with the desired estimate (3.3). This completes the Proof of Theorem 3.1.

\section{An Auxiliary optimal CONTRol Problem}

In this section, as a preliminary, we analyze an auxiliary optimal control problem. Some ideas are taken from ([6], pp. 190-199 and [4], Prop. 6.1).

Let $y \in L_{\mathbb{F}}^{2}\left(\Omega ; C\left([0, T] ; L^{2}(G)\right)\right)$ satisfy $y(0)=y(T)=0$ in $G$, P -a.s. For any $K>1$, let $\varrho \equiv \varrho^{K}(x) \in C^{2}(\bar{G})$, such that $\min _{x \in \bar{G}} \varrho(x)=1$ and

$$
\varrho(x)= \begin{cases}1 & \text { if } x \in G_{0} \\ K & \text { if } \operatorname{dist}\left(x, G_{0}\right) \geq \frac{1}{\ln K} .\end{cases}
$$

For any integer $m \geq 3$, let $h=\frac{T}{m}$, and set

$$
y_{m}^{j} \equiv y_{m}^{j}(x)=y(j h, x) \quad \text { and } \quad \phi_{m}^{j} \equiv \phi_{m}^{j}(x)=\phi(j h, x), \quad j=0,1, \ldots, m,
$$


where $\phi(t, x)=\mathrm{d}(x)-c_{1}(t-T / 2)^{2}$ (see (3.2)). Consider the following system:

$$
\begin{cases}\mathbb{E}\left(\frac{z_{m}^{j+1}-2 z_{m}^{j}+z_{m}^{j-1}}{h^{2}} \mid \mathcal{F}_{j h}\right)-\sum_{j_{1}, j_{2}=1}^{n} \partial_{x_{j_{2}}}\left(b^{j_{1} j_{2}} \partial_{x_{j_{1}}} z_{m}^{j}\right) & \\ \quad=\mathbb{E}\left(\frac{r_{1 m}^{j+1}-r_{1 m}^{j}}{h} \mid \mathcal{F}_{j h}\right)+r_{2 m}^{j}+\lambda y_{m}^{j} \mathrm{e}^{2 \lambda \phi_{m}^{j}}+r_{m}^{j}(1 \leq j \leq m-1) & \text { in } G, \\ z_{m}^{j}=0 \quad(0 \leq j \leq m) & \text { on } \Gamma, \\ z_{m}^{0}=z_{m}^{m}=r_{2 m}^{0}=r_{2 m}^{m}=r_{m}^{0}=r_{m}^{m}=0, \quad r_{1 m}^{0}=r_{1 m}^{1} & \text { in } G .\end{cases}
$$

Here $\left(r_{1 m}^{j}, r_{2 m}^{j}, r_{m}^{j}\right) \in\left(L_{\mathcal{F}_{j h}}^{2}\left(\Omega ; L^{2}(G)\right)\right)^{3}(j=0,1, \ldots, m)$ are controls. The set of admissible sequences for (4.3) is defined by

$$
\begin{aligned}
\mathcal{A}_{a d}=\left\{\left\{\left(z_{m}^{j}, r_{1 m}^{j}, r_{2 m}^{j}, r_{m}^{j}\right)\right\}_{j=0}^{m}:\right. & \left(z_{m}^{j}, r_{1 m}^{j}, r_{2 m}^{j}, r_{m}^{j}\right) \in L_{\mathcal{F}_{j h}}^{2}\left(\Omega ; H_{0}^{1}(G)\right) \times\left(L_{\mathcal{F}_{j h}}^{2}\left(\Omega ; L^{2}(G)\right)\right)^{3} \\
& \text { and } \left.\left\{\left(z_{m}^{j}, r_{1 m}^{j}, r_{2 m}^{j}, r_{m}^{j}\right)\right\}_{j=0}^{m} \text { solves }(4.3)\right\} .
\end{aligned}
$$

Since $\left\{\left(0,0,0,-\lambda y_{m}^{j} \mathrm{e}^{2 \lambda \phi_{m}^{j}}\right)\right\}_{j=0}^{m} \in \mathcal{A}_{a d}$, it follows that $\mathcal{A}_{a d} \neq \emptyset$.

Next, define a cost functional as follows:

$$
\begin{aligned}
J\left(\left\{\left(z_{m}^{j}, r_{1 m}^{j}, r_{2 m}^{j}, r_{m}^{j}\right)\right\}_{j=0}^{m}=\right. & \frac{h}{2} \mathbb{E} \int_{G} \varrho \frac{\left|r_{1 m}^{m}\right|^{2}}{\lambda^{2}} \mathrm{e}^{-2 \lambda \phi_{m}^{m}} \mathrm{~d} x+\frac{h}{2} \mathbb{E} \sum_{j=1}^{m-1}\left[\int_{G}\left|z_{m}^{j}\right|^{2} \mathrm{e}^{-2 \lambda \phi_{m}^{j}} \mathrm{~d} x\right. \\
& \left.+\int_{G} \varrho\left(\frac{\left|r_{1 m}^{j}\right|^{2}}{\lambda^{2}}+\frac{\left|r_{2 m}^{j}\right|^{2}}{\lambda^{4}}\right) \mathrm{e}^{-2 \lambda \phi_{m}^{j}} \mathrm{~d} x+K \int_{G}\left|r_{m}^{j}\right|^{2} \mathrm{~d} x\right],
\end{aligned}
$$

and consider the following optimal control problem: find a $\left\{\left(\hat{z}_{m}^{j}, \hat{r}_{1 m}^{j}, \hat{r}_{2 m}^{j}, \hat{r}_{m}^{j}\right)\right\}_{j=0}^{m} \in \mathcal{A}_{a d}$, such that

$$
J\left(\left\{\left(\hat{z}_{m}^{j}, \hat{r}_{1 m}^{j}, \hat{r}_{2 m}^{j}, \hat{r}_{m}^{j}\right)\right\}_{j=0}^{m}\right)=\min _{\left\{\left(z_{m}^{j}, r_{1 m}^{j}, r_{2 m}^{j}, r_{m}^{j}\right)\right\}_{j=0}^{m} \in \mathcal{A}_{a d}} J\left(\left\{\left(z_{m}^{j}, r_{1 m}^{j}, r_{2 m}^{j}, r_{m}^{j}\right)\right\}_{j=0}^{m}\right) .
$$

Notice that for any $\left\{\left(z_{m}^{j}, r_{1 m}^{j}, r_{2 m}^{j}, r_{m}^{j}\right)\right\}_{j=0}^{m} \in \mathcal{A}_{a d}$, by the standard regularity results for elliptic equations, we have that $z_{m}^{j} \in L_{\mathcal{F}_{T}}^{2}\left(\Omega ; H^{2}(G) \cap H_{0}^{1}(G)\right)$.

We have the following result.

Proposition 4.1. For any $K>1$ and $m \geq 3$, the problem (4.5) admits a unique solution $\left\{\left(\hat{z}_{m}^{j}, \hat{r}_{1 m}^{j}, \hat{r}_{2 m}^{j}, \hat{r}_{m}^{j}\right)\right\}_{j=0}^{m} \in \mathcal{A}_{a d}$, (which depends on $\left.K\right)$. Furthermore, define

$$
p_{m}^{j} \equiv p_{m}^{j}(x) \triangleq K \hat{r}_{m}^{j}(x), \quad 0 \leq j \leq m .
$$

Then,

$$
\left\{\begin{array}{l}
\hat{z}_{m}^{0}=\hat{z}_{m}^{m}=p_{m}^{0}=p_{m}^{m}=0 \text { in } G, \\
\hat{z}_{m}^{j}, p_{m}^{j} \in L_{\mathcal{F}_{j h}}^{2}\left(\Omega ; H^{2}(G) \cap H_{0}^{1}(G)\right), \quad 1 \leq j \leq m-1 .
\end{array}\right.
$$

Also, the following optimality conditions hold:

$$
\left\{\begin{array}{ll}
\frac{p_{m}^{j}-p_{m}^{j-1}}{h}+\varrho \frac{\hat{r}_{1 m}^{j}}{\lambda^{2}} \mathrm{e}^{-2 \lambda \phi_{m}^{j}}=0 & \text { in } G, \\
p_{m}^{j}-\varrho \frac{\hat{r}_{2 m}^{j}}{\lambda^{4}} \mathrm{e}^{-2 \lambda \phi_{m}^{j}}=0 & \text { in } G,
\end{array} \quad 1 \leq j \leq m,\right.
$$




$$
\begin{cases}\mathbb{E}\left(\frac{p_{m}^{j+1}-2 p_{m}^{j}+p_{m}^{j-1}}{h^{2}} \mid \mathcal{F}_{j h}\right)-\sum_{j_{1}, j_{2}=1}^{n} \partial_{x_{j_{2}}}\left(b^{j_{1} j_{2}} \partial_{x_{j_{1}}} p_{m}^{j}\right)+\mathrm{e}^{-2 \lambda \phi_{m}^{j}} \hat{z}_{m}^{j}=0 & \text { in } G, \quad 1 \leq j \leq m-1 . \\ p_{m}^{j}=0 & \text { on } \Gamma,\end{cases}
$$

Moreover, there is a constant $C=C(K, \lambda)>0$, independent of $m$, such that

$$
h \mathbb{E} \sum_{j=1}^{m-1} \int_{G}\left[\left|\hat{z}_{m}^{j}\right|^{2}+\left|\hat{r}_{1 m}^{j}\right|^{2}+\left|\hat{r}_{2 m}^{j}\right|^{2}+K\left|\hat{r}_{m}^{j}\right|^{2}\right] \mathrm{d} x+h \mathbb{E} \int_{G}\left|\hat{r}_{1 m}^{m}\right|^{2} \mathrm{~d} x \leq C,
$$

and

$$
h \mathbb{E} \sum_{j=0}^{m-1} \int_{G}\left\{\frac{\left(\hat{z}_{m}^{j+1}-\hat{z}_{m}^{j}\right)^{2}}{h^{2}}+\frac{\left[\mathbb{E}\left(\hat{r}_{1 m}^{j+1}-\hat{r}_{1 m}^{j} \mid \mathcal{F}_{j h}\right)\right]^{2}}{h^{2}}+\frac{\left(\hat{r}_{2 m}^{j+1}-\hat{r}_{2 m}^{j}\right)^{2}}{h^{2}}+K \frac{\left(\hat{r}_{m}^{j+1}-\hat{r}_{m}^{j}\right)^{2}}{h^{2}}\right\} \mathrm{d} x \leq C .
$$

We refer to Appendix A for a proof of this proposition.

\section{Global Carleman estimate for stochastic hyperbolic equations IN THE $L^{2}$-SPACE}

We define a formal differential operator $\mathcal{A}$ by

$$
\mathcal{A} \triangleq \frac{\partial^{2}}{\partial t^{2}}-\sum_{i, j=1}^{n} \frac{\partial}{\partial x_{j}}\left(b^{i j} \frac{\partial}{\partial x_{i}}\right) .
$$

In order to prove Theorem 1.6, we need the following global Carleman estimate for stochastic hyperbolic equations.

Theorem 5.1. Assume that the Condition 1.2 holds. Let $T_{0}$ be given by (1.14). Then there exists a $\lambda_{0}^{*}>0$ such that for any $T>T_{0}, \lambda \geq \lambda_{0}^{*}$, and any $y \in L_{\mathbb{F}}^{2}\left(\Omega ; C\left([0, T] ; L^{2}(G)\right)\right)$ satisfying $y(0)=y(T)=0$ in $G$ and

$$
\mathbb{E}(y, \mathcal{A} \eta)_{L^{2}(Q)}=\mathbb{E}\left\langle b_{1} y+f, \eta\right\rangle_{H^{-1}(Q), H_{0}^{1}(Q)}, \quad \forall \eta \in L_{\mathbb{F}}^{2}\left(\Omega ; H_{0}^{1}(Q)\right) \text { with } \mathcal{A} \eta \in L_{\mathbb{F}}^{2}\left(0, T ; L^{2}(G)\right),
$$

it holds that

$$
\lambda \mathbb{E} \int_{Q} \mathrm{e}^{2 \lambda \phi} y^{2} \mathrm{~d} x \mathrm{~d} t \leq C\left(\mathbb{E}\left|\mathrm{e}^{\lambda \phi} f\right|_{H^{-1}(Q)}^{2}+\left|\mathrm{e}^{\lambda \phi} b_{1} y\right|_{L_{\mathbb{F}}^{2}\left(0, T ; H^{-1}(G)\right)}^{2}+\lambda^{2} \mathbb{E} \int_{0}^{T} \int_{G_{0}} \mathrm{e}^{2 \lambda \phi} y^{2} \mathrm{~d} x \mathrm{~d} t\right) .
$$

Proof of Theorem 5.1. We borrow some idea from [4,6,10]. The whole proof is divided into six steps.

Step 1. First, recall the functions $\left\{\left(\hat{z}_{m}^{j}, \hat{r}_{1 m}^{j}, \hat{r}_{2 m}^{j}, \hat{r}_{m}^{j}\right)\right\}_{j=0}^{m}$ in Proposition 4.1. For $m=2^{i}(i=2,3, \ldots)$, we define

$$
\left\{\begin{array}{l}
\tilde{z}^{m}(t, x)=\frac{1}{h} \sum_{j=0}^{m-1} \mathbb{E}\left(\left\{(t-j h) \hat{z}_{m}^{j+1}(x)-[t-(j+1) h] \hat{z}_{m}^{j}(x)\right\} \mid \mathcal{F}_{j h}\right) \chi_{(j h,(j+1) h]}(t), \\
\tilde{r}_{1}^{m}(t, x)=\frac{1}{h} \sum_{j=0}^{m-1} \mathbb{E}\left(\left\{(t-j h) \hat{r}_{1 m}^{j+1}(x)-[t-(j+1) h] \hat{r}_{1 m}^{j}(x)\right\} \mid \mathcal{F}_{j h}\right) \chi_{(j h,(j+1) h]}(t), \\
\tilde{r}_{2}^{m}(t, x)=\frac{1}{h} \sum_{j=0}^{m-1} \mathbb{E}\left(\left\{(t-j h) \hat{r}_{2 m}^{j+1}(x)-[t-(j+1) h] \hat{r}_{2 m}^{j}(x)\right\} \mid \mathcal{F}_{j h}\right) \chi_{(j h,(j+1) h]}(t), \\
\tilde{r}^{m}(t, x)=\frac{1}{h} \sum_{j=0}^{m-1} \mathbb{E}\left(\left\{(t-j h) \hat{r}_{m}^{j+1}(x)-[t-(j+1) h] \hat{r}_{m}^{j}(x)\right\} \mid \mathcal{F}_{j h}\right) \chi_{(j h,(j+1) h]}(t) .
\end{array}\right.
$$


By (4.10) and (4.11), there is a subsequence of $\left\{\left(\tilde{z}^{m}, \tilde{r}_{1}^{m}, \tilde{r}_{2}^{m}, \tilde{r}^{m}\right)\right\}_{m=2}^{\infty}$ (still denoted by itself), such that for some $\left(\tilde{z}, \tilde{r}_{1}, \tilde{r}_{2}, \tilde{r}\right) \in\left(L_{\mathbb{F}}^{2}\left(\Omega ; H^{1}\left(0, T ; L^{2}(G)\right)\right)\right)^{4}$, as $m \rightarrow \infty$,

$$
\left(\tilde{z}^{m}, \tilde{r}_{1}^{m}, \tilde{r}_{2}^{m}, \tilde{r}^{m}\right) \rightarrow\left(\tilde{z}, \tilde{r}_{1}, \tilde{r}_{2}, \tilde{r}\right) \text { weakly in }\left(L_{\mathbb{F}}^{2}\left(\Omega ; H^{1}\left(0, T ; L^{2}(G)\right)\right)\right)^{4} .
$$

Also, by (4.3), $\tilde{z} \in L_{\mathbb{F}}^{2}\left(\Omega ; H^{1}\left(0, T ; L^{2}(G)\right)\right)$ is the weak solution to the following random hyperbolic equation:

$$
\begin{cases}\mathcal{A} \tilde{z}=\tilde{r}_{1, t}+\tilde{r}_{2}+\lambda y \mathrm{e}^{2 \lambda \phi}+\tilde{r} & \text { in } Q, \\ \tilde{z}=0 & \text { on } \Sigma, \\ \tilde{z}(0)=\tilde{z}(T)=0 & \text { in } G .\end{cases}
$$

This implies that

$$
\tilde{z} \in L_{\mathbb{F}}^{2}\left(\Omega ; C\left([0, T] ; H_{0}^{1}(\Omega)\right)\right) \cap L_{\mathbb{F}}^{2}\left(\Omega ; C^{1}\left([0, T] ; L^{2}(\Omega)\right)\right) .
$$

The proof of (5.6) is given in the Appendix B. For any constant $K>1$, put

$$
\tilde{p} \triangleq K \tilde{r} .
$$

By (4.8)-(4.11), it is easy to see that $\tilde{p}$ is the solution to the following system:

$$
\begin{cases}\mathcal{A} \tilde{p}+\tilde{z} \mathrm{e}^{-2 \lambda \phi}=0 & \text { in } Q, \\ \tilde{p}=0 & \text { on } \Sigma \\ \tilde{p}(0)=\tilde{p}(T)=0 & \text { in } G \\ \tilde{p}_{t}+\varrho \frac{\tilde{r}_{1}}{\lambda^{2}} \mathrm{e}^{-2 \lambda \phi}=0 & \text { in } Q \\ \tilde{p}-\varrho \frac{\tilde{r}_{2}}{\lambda^{4}} \mathrm{e}^{-2 \lambda \phi}=0 & \text { in } Q\end{cases}
$$

Noting that $\left(\tilde{r}_{1}, \tilde{r}_{2}\right) \in\left(L_{\mathbb{F}}^{2}\left(\Omega ; H^{1}\left(0, T ; L^{2}(G)\right)\right)\right)^{2}$, similar to the proof of $(5.6)$, we can also deduce that

$$
\tilde{p} \in L_{\mathbb{F}}^{2}\left(\Omega ; C\left([0, T] ; H_{0}^{1}(G)\right)\right) \cap L_{\mathbb{F}}^{2}\left(\Omega ; C^{1}\left([0, T] ; L^{2}(G)\right)\right) .
$$

Step 2. Applying Theorem 3.1 to $\tilde{p}$ in (5.7), we obtain that

$$
\begin{aligned}
\lambda \mathbb{E} \int_{Q}\left(\lambda^{2} \tilde{p}^{2}+\tilde{p}_{t}^{2}+|\nabla \tilde{p}|^{2}\right) \mathrm{e}^{2 \lambda \phi} \mathrm{d} x \mathrm{~d} t & \leq C\left[\mathbb{E} \int_{Q} \tilde{z}^{2} \mathrm{e}^{-2 \lambda \phi} \mathrm{d} x \mathrm{~d} t+\lambda^{2} \mathbb{E} \int_{0}^{T} \int_{G_{0}}\left(\lambda^{2} \tilde{p}^{2}+\tilde{p}_{t}^{2}\right) \mathrm{e}^{2 \lambda \phi} \mathrm{d} x \mathrm{~d} t\right] \\
& \leq C\left[\mathbb{E} \int_{Q} \tilde{z}^{2} \mathrm{e}^{-2 \lambda \phi} \mathrm{d} x \mathrm{~d} t+\mathbb{E} \int_{0}^{T} \int_{G_{0}}\left(\frac{\tilde{r}_{1}^{2}}{\lambda^{2}}+\frac{\tilde{r}_{2}^{2}}{\lambda^{4}}\right) \mathrm{e}^{-2 \lambda \phi} \mathrm{d} x \mathrm{~d} t\right] .
\end{aligned}
$$

Here and hereafter, $C$ denotes a constant, independent of $K$ and $\lambda$. Moreover, by (5.7) again, $\tilde{p}_{t}$ satisfies

$$
\begin{cases}\mathcal{A} \tilde{p}_{t}+\left(\tilde{z} \mathrm{e}^{-2 \lambda \phi}\right)_{t}=0 & \text { in } Q \\ \tilde{p}_{t}=0 & \text { on } \Sigma \\ \tilde{p}_{t t}+\frac{\varrho}{\lambda}\left(\frac{\tilde{r}_{1, t}}{\lambda}-2 \phi_{t} \tilde{r}_{1}\right) \mathrm{e}^{-2 \lambda \phi}=0 & \text { in } Q \\ \tilde{p}_{t}-\frac{\varrho}{\lambda^{2}}\left(\frac{\tilde{r}_{2, t}}{\lambda^{2}}-\frac{2}{\lambda} \phi_{t} \tilde{r}_{2}\right) \mathrm{e}^{-2 \lambda \phi}=0 & \text { in } Q\end{cases}
$$


Applying Theorem 3.1 to $\tilde{p}_{t}$, by (5.9), we obtain that

$$
\begin{aligned}
& \lambda \mathbb{E} \int_{Q}\left(\lambda^{2} \tilde{p}_{t}^{2}+\tilde{p}_{t t}^{2}+\left|\nabla \tilde{p}_{t}\right|^{2}\right) \mathrm{e}^{2 \lambda \phi} \mathrm{d} x \mathrm{~d} t \\
& \leq C\left[\mathbb{E}\left|\mathrm{e}^{\lambda \phi}\left(\mathrm{e}^{-2 \lambda \phi} \tilde{z}\right)_{t}\right|_{L^{2}(Q)}^{2}+\lambda^{2} \mathbb{E} \int_{0}^{T} \int_{G_{0}}\left(\lambda^{2} \tilde{p}_{t}^{2}+\tilde{p}_{t t}^{2}\right) \mathrm{e}^{2 \lambda \phi} \mathrm{d} x \mathrm{~d} t\right] \\
& \leq C\left[\mathbb{E} \int_{Q}\left(\tilde{z}_{t}^{2}+\lambda^{2} \tilde{z}^{2}\right) \mathrm{e}^{-2 \lambda \phi} \mathrm{d} x \mathrm{~d} t+\mathbb{E} \int_{0}^{T} \int_{G_{0}}\left(\frac{\tilde{r}_{1, t}^{2}}{\lambda^{2}}+\frac{\tilde{r}_{2, t}^{2}}{\lambda^{4}}+\tilde{r}_{1}^{2}+\frac{\tilde{r}_{2}^{2}}{\lambda^{2}}\right) \mathrm{e}^{-2 \lambda \phi} \mathrm{d} x \mathrm{~d} t\right] .
\end{aligned}
$$

Step 3. By (5.7), we have that

$$
-\mathbb{E} \int_{Q}\left(\tilde{r}_{1, t}+\tilde{r}_{2}\right) \tilde{p} \mathrm{~d} x \mathrm{~d} t=\mathbb{E} \int_{Q}\left(\tilde{r}_{1} \tilde{p}_{t}-\tilde{r}_{2} \tilde{p}\right) \mathrm{d} x \mathrm{~d} t=-\mathbb{E} \int_{Q} \varrho\left(\frac{\tilde{r}_{1}^{2}}{\lambda^{2}}+\frac{\tilde{r}_{2}^{2}}{\lambda^{4}}\right) \mathrm{e}^{-2 \lambda \phi} \mathrm{d} x \mathrm{~d} t .
$$

This implies that

$$
\begin{aligned}
& 0=\mathbb{E}\left(\mathcal{A} \tilde{z}-\tilde{r}_{1, t}-\tilde{r}_{2}-\lambda y \mathrm{e}^{2 \lambda \phi}-\tilde{r}, \tilde{p}\right)_{L^{2}(Q)} \\
& =-\mathbb{E} \int_{Q} \tilde{z}^{2} \mathrm{e}^{-2 \lambda \phi} \mathrm{d} x \mathrm{~d} t-\mathbb{E} \int_{Q} \varrho\left(\frac{\tilde{r}_{1}^{2}}{\lambda^{2}}+\frac{\tilde{r}_{2}^{2}}{\lambda^{4}}\right) \mathrm{e}^{-2 \lambda \phi} \mathrm{d} x \mathrm{~d} t-\mathbb{E} \lambda \int_{Q} y \tilde{p} \mathrm{e}^{2 \lambda \phi} \mathrm{d} x \mathrm{~d} t-K \mathbb{E} \int_{Q} \tilde{r}^{2} \mathrm{~d} x \mathrm{~d} t .
\end{aligned}
$$

Hence,

$$
\mathbb{E} \int_{Q} \tilde{z}^{2} \mathrm{e}^{-2 \lambda \phi} \mathrm{d} x \mathrm{~d} t+\mathbb{E} \int_{Q} \varrho\left(\frac{\tilde{r}_{1}^{2}}{\lambda^{2}}+\frac{\tilde{r}_{2}^{2}}{\lambda^{4}}\right) \mathrm{e}^{-2 \lambda \phi} \mathrm{d} x \mathrm{~d} t+K \mathbb{E} \int_{Q} \tilde{r}^{2} \mathrm{~d} x \mathrm{~d} t=-\lambda \mathbb{E} \int_{Q} y \tilde{p} \mathrm{e}^{2 \lambda \phi} \mathrm{d} x \mathrm{~d} t .
$$

Combining (5.8) and (5.11), we arrive at

$$
\mathbb{E} \int_{Q} \tilde{z}^{2} \mathrm{e}^{-2 \lambda \phi} \mathrm{d} x \mathrm{~d} t+\mathbb{E} \int_{Q} \varrho\left(\frac{\tilde{r}_{1}^{2}}{\lambda^{2}}+\frac{\tilde{r}_{2}^{2}}{\lambda^{4}}\right) \mathrm{e}^{-2 \lambda \phi} \mathrm{d} x \mathrm{~d} t+K \mathbb{E} \int_{Q} \tilde{r}^{2} \mathrm{~d} x \mathrm{~d} t \leq \frac{C}{\lambda} \mathbb{E} \int_{Q} y^{2} \mathrm{e}^{2 \lambda \phi} \mathrm{d} x \mathrm{~d} t .
$$

Step 4. Using (5.5) and (5.9) again, and noting $\tilde{p}_{t t}(0)=\tilde{p}_{t t}(T)=0$ in $G$, we find that

$$
\begin{aligned}
0 & =\mathbb{E}\left(\mathcal{A} \tilde{z}-\tilde{r}_{1, t}-\tilde{r}_{2}-\lambda y \mathrm{e}^{2 \lambda \phi}-\tilde{r}, \tilde{p}_{t t}\right)_{L^{2}(Q)} \\
& =-\mathbb{E} \int_{Q} \tilde{z}\left(\mathrm{e}^{-2 \lambda \phi} \tilde{z}\right)_{t t} \mathrm{~d} x \mathrm{~d} t-\mathbb{E} \int_{Q}\left(\tilde{r}_{1, t}+\tilde{r}_{2}\right) \tilde{p}_{t t} \mathrm{~d} x \mathrm{~d} t-\lambda \mathbb{E} \int_{Q} y \tilde{p}_{t t} \mathrm{e}^{2 \lambda \phi} \mathrm{d} x \mathrm{~d} t-\mathbb{E} \int_{Q} \tilde{r} \tilde{p}_{t t} \mathrm{~d} x \mathrm{~d} t .
\end{aligned}
$$

Notice that

$$
-\mathbb{E} \int_{Q} \tilde{z}\left(\mathrm{e}^{-2 \lambda \phi} \tilde{z}\right)_{t t} \mathrm{~d} x \mathrm{~d} t=\mathbb{E} \int_{Q}\left[\tilde{z}_{t}^{2} \mathrm{e}^{-2 \lambda \phi} \mathrm{d} x \mathrm{~d} t-\frac{\tilde{z}^{2}}{2}\left(\mathrm{e}^{-2 \lambda \phi}\right)_{t t}\right] \mathrm{d} x \mathrm{~d} t=\mathbb{E} \int_{Q}\left(\tilde{z}_{t}^{2}+\lambda \phi_{t t} \tilde{z}^{2}-2 \lambda{ }^{2} \phi_{t}^{2} \tilde{z}^{2}\right) \mathrm{e}^{-2 \lambda \phi} \mathrm{d} x \mathrm{~d} t .
$$

Further, in view of the third and fourth equalities in (5.9), it follows that

$$
\begin{aligned}
& -\mathbb{E} \int_{Q}\left(\tilde{r}_{1, t}+\tilde{r}_{2}\right) \tilde{p}_{t t} \mathrm{~d} x \mathrm{~d} t=-\mathbb{E} \int_{Q}\left(\tilde{r}_{1, t} \tilde{p}_{t t}-\tilde{r}_{2, t} \tilde{p}_{t}\right) \mathrm{d} x \mathrm{~d} t \\
& =\mathbb{E} \int_{Q} \tilde{r}_{1, t} \frac{\varrho}{\lambda}\left(\frac{\tilde{r}_{1, t}}{\lambda}-2 \phi_{t} \tilde{r}_{1}\right) \mathrm{e}^{-2 \lambda \phi} \mathrm{d} x \mathrm{~d} t+\mathbb{E} \int_{Q} \tilde{r}_{2, t} \frac{\varrho}{\lambda^{2}}\left(\frac{\tilde{r}_{2, t}}{\lambda^{2}}-\frac{2}{\lambda} \phi_{t} \tilde{r}_{2}\right) \mathrm{e}^{-2 \lambda \phi} \mathrm{d} x \mathrm{~d} t \\
& =\mathbb{E} \int_{Q} \varrho\left(\frac{\tilde{r}_{1, t}^{2}}{\lambda^{2}}+\frac{\tilde{r}_{2, t}^{2}}{\lambda^{4}}-\frac{2}{\lambda} \phi_{t} \tilde{r}_{1} \tilde{r}_{1, t}-\frac{2}{\lambda^{3}} \phi_{t} \tilde{r}_{2} \tilde{r}_{2, t}\right) \mathrm{e}^{-2 \lambda \phi} \mathrm{d} x \mathrm{~d} t .
\end{aligned}
$$


Further, by $\tilde{p}=K \tilde{r}$ and integration by parts,

$$
-\mathbb{E} \int_{Q} \tilde{r} \tilde{p}_{t t} \mathrm{~d} x \mathrm{~d} t=K \mathbb{E} \int_{Q} \tilde{r}_{t}^{2} \mathrm{~d} x \mathrm{~d} t
$$

Therefore, by (5.13)-(5.16), we get that

$$
\begin{aligned}
& \mathbb{E} \int_{Q} \varrho\left(\frac{\tilde{r}_{1, t}^{2}}{\lambda^{2}}+\frac{\tilde{r}_{2, t}^{2}}{\lambda^{4}}-\frac{2}{\lambda} \phi_{t} \tilde{r}_{1} \tilde{r}_{1, t}-\frac{2}{\lambda^{3}} \phi_{t} \tilde{r}_{2} \tilde{r}_{2, t}\right) \mathrm{e}^{-2 \lambda \phi} \mathrm{d} x \mathrm{~d} t+K \mathbb{E} \int_{Q} \tilde{r}_{t}^{2} \mathrm{~d} x \mathrm{~d} t \\
& +\mathbb{E} \int_{Q}\left(\tilde{z}_{t}^{2}+\lambda \phi_{t t} \tilde{z}^{2}-2 \lambda^{2} \phi_{t}^{2} \tilde{z}^{2}\right) \mathrm{e}^{-2 \lambda \phi} \mathrm{d} x \mathrm{~d} t=\lambda \mathbb{E} \int_{Q} y \tilde{p}_{t t} \mathrm{e}^{2 \lambda \phi} \mathrm{d} x \mathrm{~d} t .
\end{aligned}
$$

Now, by (5.17) and (5.12), using the Cauchy-Schwarz inequality and noting (5.10), we obtain that

$$
\mathbb{E} \int_{Q}\left(\tilde{z}_{t}^{2}+\lambda^{2} \tilde{z}^{2}\right) \mathrm{e}^{-2 \lambda \phi} \mathrm{d} x \mathrm{~d} t+\mathbb{E} \int_{Q} \varrho\left(\frac{\tilde{r}_{1, t}^{2}}{\lambda^{2}}+\frac{\tilde{r}_{2, t}^{2}}{\lambda^{4}}+\tilde{r}_{1}^{2}+\frac{\tilde{r}_{2}^{2}}{\lambda^{2}}\right) \mathrm{e}^{-2 \lambda \phi} \mathrm{d} x \mathrm{~d} t \leq C \lambda \mathbb{E} \int_{Q} y^{2} \mathrm{e}^{2 \lambda \phi} \mathrm{d} x \mathrm{~d} t .
$$

Step 5. By (5.7), we find that

$$
\begin{aligned}
& \mathbb{E}\left(\tilde{r}_{1, t}+\tilde{r}_{2}+\lambda y \mathrm{e}^{2 \lambda \phi}+\tilde{r}, \tilde{z} \mathrm{e}^{-2 \lambda \phi}\right)_{L^{2}(Q)}=\mathbb{E}\left(\mathcal{A} \tilde{z}, \tilde{z} \mathrm{e}^{-2 \lambda \phi}\right)_{L^{2}(Q)} \\
= & -\mathbb{E} \int_{Q} \tilde{z}_{t}\left(\tilde{z} \mathrm{e}^{-2 \lambda \phi}\right)_{t} \mathrm{~d} x \mathrm{~d} t+\sum_{j, k=1}^{n} \mathbb{E} \int_{Q} b^{j k} \tilde{z}_{x_{j}}\left(\tilde{z} \mathrm{e}^{-2 \lambda \phi}\right)_{x_{k}} \mathrm{~d} x \mathrm{~d} t \\
= & -\mathbb{E} \int_{Q}\left(\tilde{z}_{t}^{2}+\lambda \phi_{t t} \tilde{z}^{2}-2 \lambda^{2} \phi_{t}^{2} \tilde{z}^{2}\right) \mathrm{e}^{-2 \lambda \phi} \mathrm{d} x \mathrm{~d} t+\sum_{j, k=1}^{n} \mathbb{E} \int_{Q} b^{j k} \tilde{z}_{x_{j}} \tilde{z}_{x_{k}} \mathrm{e}^{-2 \lambda \phi} \mathrm{d} x \mathrm{~d} t \\
& -2 \lambda \sum_{j, k=1}^{n} \mathbb{E} \int_{Q} b^{j k} \tilde{z}_{x_{j}} \tilde{z} \phi_{x_{k}} \mathrm{e}^{-2 \lambda \phi} \mathrm{d} x \mathrm{~d} t
\end{aligned}
$$

This yields that

$$
\begin{aligned}
\mathbb{E} \int_{Q}|\nabla \tilde{z}|^{2} \mathrm{e}^{-2 \lambda \phi} \mathrm{d} x \mathrm{~d} t & \leq C \mathbb{E} \int_{Q}\left[\left|\tilde{r}_{1, t}+\tilde{r}_{2}+\tilde{r}\right||\tilde{z}| \mathrm{e}^{-2 \lambda \phi}+\lambda|y \tilde{z}|+\left(\tilde{z}_{t}^{2}+\lambda^{2} \tilde{z}^{2}\right) \mathrm{e}^{-2 \lambda \phi}\right] \mathrm{d} x \mathrm{~d} t \\
& \leq C \mathbb{E} \int_{Q}\left[y^{2} \mathrm{e}^{2 \lambda \phi}+\left(\frac{\tilde{r}_{1, t}^{2}}{\lambda^{2}}+\frac{\tilde{r}_{2}^{2}}{\lambda^{2}}+\tilde{r}^{2}+\tilde{z}_{t}^{2}+\lambda^{2} \tilde{z}^{2}\right) \mathrm{e}^{-2 \lambda \phi}\right] \mathrm{d} x \mathrm{~d} t .
\end{aligned}
$$

By (5.12), (5.18) and (5.20), we choose a constant $K$ in (5.12) so that

$$
K \geq C \mathrm{e}^{2 \lambda} \max _{(t, x) \in Q}|\phi|
$$

(to absorb the term $C \mathbb{E} \int_{Q} \check{r}^{2} \mathrm{e}^{-2 \lambda \phi} \mathrm{d} x \mathrm{~d} t$ in (5.20)). Then we deduce that

$$
\mathbb{E} \int_{Q}\left(|\nabla \tilde{z}|^{2}+\tilde{z}_{t}^{2}+\lambda^{2} \tilde{z}^{2}\right) \mathrm{e}^{-2 \lambda \phi} \mathrm{d} x \mathrm{~d} t+\mathbb{E} \int_{Q} \varrho\left(\frac{\tilde{r}_{1, t}^{2}}{\lambda^{2}}+\frac{\tilde{r}_{2, t}^{2}}{\lambda^{4}}+\tilde{r}_{1}^{2}+\frac{\tilde{r}_{2}^{2}}{\lambda^{2}}\right) \mathrm{e}^{-2 \lambda \phi} \mathrm{d} x \mathrm{~d} t \leq C \lambda \mathbb{E} \int_{Q} y^{2} \mathrm{e}^{2 \lambda \phi} \mathrm{d} x \mathrm{~d} t .
$$

Step 6. Recall that $\left(\tilde{z}, \tilde{r}_{1}, \tilde{r}_{2}, \tilde{r}\right)$ depend on $K$. Now, we fix $\lambda$ and let $K$ tend to infinity. By (5.12) and (5.22), we conclude that there exists a subsequence of

$$
\begin{aligned}
\left(\tilde{z}, \tilde{r}_{1}, \tilde{r}_{2}, \tilde{r}\right) \in & \left(L_{\mathbb{F}}^{2}\left(\Omega ; H_{0}^{1}\left(0, T ; L^{2}(G)\right)\right) \cap L_{\mathbb{F}}^{2}\left(\Omega ; L^{2}\left(0, T ; H_{0}^{1}(G)\right)\right)\right) \\
& \times\left(L_{\mathbb{F}}^{2}\left(\Omega ; H^{1}\left(0, T ; L^{2}(G)\right)\right)\right)^{2} \times L_{\mathbb{F}}^{2}\left(0, T ; L^{2}(G)\right),
\end{aligned}
$$


which converges weakly to some $\left(\bar{z}, \bar{r}_{1}, \bar{r}_{2}, 0\right)$, with $\operatorname{supp} \bar{r}_{j} \subset \overline{(0, T) \times G_{0}}(j=1,2)$, since $\varrho(x) \equiv \varrho^{K}(x) \rightarrow \infty$ for any $x \notin G_{0}$, as $K \rightarrow \infty$. By (5.7), we deduce that $\left(\bar{z}, \bar{r}_{1}, \bar{r}_{2}\right)$ satisfies

$$
\begin{cases}\mathcal{A} \bar{z}=\bar{r}_{1, t}+\bar{r}_{2}+\lambda y \mathrm{e}^{2 \lambda \phi} & \text { in } Q \\ \bar{z}=0 & \text { on } \partial Q .\end{cases}
$$

Using (5.22) again, we find that

$$
\mathbb{E}\left|\bar{z} \mathrm{e}^{-\lambda \phi}\right|_{H_{0}^{1}(Q)}^{2}+\frac{1}{\lambda^{2}} \mathbb{E} \int_{0}^{T} \int_{G_{0}}\left(\bar{r}_{1, t}^{2}+\bar{r}_{2}^{2}\right) \mathrm{e}^{-2 \lambda \phi} \mathrm{d} x \mathrm{~d} t \leq C \lambda \mathbb{E} \int_{Q} y^{2} \mathrm{e}^{2 \lambda \phi} \mathrm{d} x \mathrm{~d} t .
$$

By (5.1), with $\eta$ replaced by $\bar{z}$ above, one gets that

$$
\mathbb{E}\left(y, \bar{r}_{1, t}+\bar{r}_{2}+\lambda y \mathrm{e}^{2 \lambda \phi}\right)_{L^{2}(Q)}=\mathbb{E}\left(b_{1} y+f, \bar{z}\right)_{L^{2}(Q)} .
$$

Hence, for any $\varepsilon>0$,

$$
\begin{aligned}
\lambda \mathbb{E} \int_{Q} y^{2} \mathrm{e}^{2 \lambda \phi} \mathrm{d} x \mathrm{~d} t= & \mathbb{E}(f, \bar{z})_{L^{2}(Q)}+\mathbb{E}\left(b_{1} y, \bar{z}\right)_{L^{2}(Q)}-\mathbb{E}\left(y, \bar{r}_{1, t}+\bar{r}_{2}\right)_{L^{2}\left((0, T) \times G_{0}\right)} \\
\leq & C\left\{\frac{1}{\varepsilon}\left[\mathbb{E}\left|\mathrm{e}^{\lambda \phi} f\right|_{H^{-1}(Q)}^{2}+\mathbb{E}\left|\mathrm{e}^{\lambda \phi} b_{1} y\right|_{L^{2}\left(0, T ; H^{-1}(G)\right)}^{2}+\lambda^{2} \mathbb{E} \int_{0}^{T} \int_{G_{0}} y^{2} \mathrm{e}^{2 \lambda \phi} \mathrm{d} x \mathrm{~d} t\right]\right. \\
& \left.+\varepsilon\left[\mathbb{E}\left|\mathrm{e}^{-\lambda \phi} \bar{z}\right|_{H_{0}^{1}(Q)}^{2}+\mathbb{E}\left|\bar{z} \mathrm{e}^{-\lambda \phi}\right|_{L^{2}\left(0, T ; H_{0}^{1}(G)\right)}^{2}+\frac{1}{\lambda^{2}} \mathbb{E} \int_{0}^{T} \int_{G_{0}}\left(\bar{r}_{1, t}^{2}+\bar{r}_{2}^{2}\right) \mathrm{e}^{-2 \lambda \phi} \mathrm{d} x \mathrm{~d} t\right]\right\} .
\end{aligned}
$$

Finally, choosing $\varepsilon$ in (5.25) sufficiently small and noting (5.24), we arrive at the desired estimate (5.2). This completes the Proof of Theorem 5.1.

\section{An ENERGy EStimate FOR BACKWARD STOCHASTIC HYPERBOLIC EQUATIONS}

In this section, we establish energy estimates respectively for a random hyperbolic equation and a backward stochastic hyperbolic equation, which will play important roles in the proof of Theorem 1.6.

First, set $\widehat{T} \in[0, T)$ and consider the following random hyperbolic equation:

$$
\begin{cases}\vartheta_{t t}-\sum_{i, j=1}^{n}\left(b^{i j} \vartheta_{x_{i}}\right)_{x_{j}} \mathrm{~d} t=b_{1} \vartheta & \text { in }(\widehat{T}, T) \times G, \\ \vartheta=0 & \text { on }(\widehat{T}, T) \times \Gamma, \\ \vartheta(\widehat{T})=\vartheta_{0}, \quad \vartheta_{t}(\widehat{T})=\vartheta_{1} & \text { in } G .\end{cases}
$$

It is easy to see that for any $\left(\vartheta_{0}, \vartheta_{1}\right) \in L^{2}\left(\Omega, \mathcal{F}_{\widehat{T}}, \mathcal{P} ; H_{0}^{1}(G)\right) \times L^{2}\left(\Omega, \mathcal{F}_{\widehat{T}}, \mathcal{P} ; L^{2}(G)\right)$, (6.1) admits a unique solution

$$
\vartheta \in L_{\mathbb{F}}^{2}\left(\Omega ; C\left([0, T] ; H_{0}^{1}(G)\right)\right) \bigcap L_{\mathbb{F}}^{2}\left(\Omega ; C^{1}\left([0, T] ; L^{2}(G)\right)\right) .
$$

Furthermore, we have the following energy estimate.

Proposition 6.1. There is a constant $C>0$, depending only on $T, G$ and $b^{i j}(1 \leq i, j \leq n)$, such that for any solution $\vartheta$ to (6.1) and for all $t, s$ satisfying $\widehat{T} \leq t \leq s \leq T$, it holds that

$$
\mathbb{E} \int_{G}\left(\left|\vartheta_{t}(s, x)\right|^{2}+|\nabla \vartheta(s, x)|^{2}\right) \mathrm{d} x \leq C \mathrm{e}^{C r_{1}^{\frac{1}{2-n / p}}(t-s)} \mathbb{E} \int_{G}\left(\left|\vartheta_{t}(t, x)\right|^{2}+|\nabla \vartheta(t, x)|^{2}\right) \mathrm{d} x .
$$


Next, let $\widetilde{T} \in(0, T]$. We consider the following backward stochastic hyperbolic equation:

$$
\begin{cases}d \alpha=\beta \mathrm{d} t+\eta \mathrm{d} B(t) & \text { in }(0, \widetilde{T}) \times G, \\ \mathrm{~d} \beta-\sum_{i, j=1}^{n}\left(b^{i j} \alpha_{x_{i}}\right)_{x_{j}} \mathrm{~d} t=b_{1} \alpha \mathrm{d} t+b_{2} \eta \mathrm{d} t+\zeta \mathrm{d} B(t) & \text { in }(0, \widetilde{T}) \times G, \\ \alpha=0, \beta=0 & \text { on }(0, \widetilde{T}) \times \Gamma, \\ \alpha(\widetilde{T})=\alpha_{0}, \quad \beta(\widetilde{T})=\beta_{0} & \text { in } G .\end{cases}
$$

Set

$$
\mathbb{H}_{\widetilde{T}}=L_{\mathbb{F}}^{2}\left(\Omega ; C\left([0, \widetilde{T}] ; H_{0}^{1}(G)\right)\right) \times L_{\mathbb{F}}^{2}\left(\Omega ; C\left([0, \widetilde{T}] ; L^{2}(G)\right)\right) \times L_{\mathbb{F}}^{2}\left(0, \widetilde{T} ; H_{0}^{1}(G)\right) \times L_{\mathbb{F}}^{2}\left(0, \widetilde{T} ; L^{2}(G)\right) .
$$

We shall use the following notion of solution for the system (6.3).

Definition 6.2. $(\alpha, \beta, \eta, \zeta) \in \mathbb{H}_{\widetilde{T}}$ is called a solution to the system (6.3), if

(1) $\alpha(\widetilde{T})=\alpha_{0}$ and $\beta(\widetilde{T})=\beta_{0}$ in $G, \mathcal{P}$-a.s.

(2) For any $t \in(0, \widetilde{T})$ and $\varphi \in C_{0}^{1}(G)$, it holds that

$$
\alpha(\widetilde{T})-\alpha(t)=\int_{t}^{\widetilde{T}} \beta(s) \mathrm{d} s+\int_{t}^{\widetilde{T}} \eta(s) \mathrm{d} B(s) \quad \text { in } G, \quad \mathcal{P} \text {-a.s. }
$$

and

$$
\begin{aligned}
\langle\beta(\widetilde{T}), \varphi\rangle_{L^{2}(G)}-\langle\beta(t), \varphi\rangle_{L^{2}(G)}= & \int_{t}^{\widetilde{T}} \int_{G}\left[-\sum_{i, j=1}^{n} b^{i j}(x) \varphi_{x_{i}}(x) \alpha_{x_{j}}(s, x)+b_{1}(s, x) \alpha(s, x) \varphi(x)\right] \mathrm{d} x \mathrm{~d} s \\
& +\int_{t}^{\widetilde{T}} \int_{G}\left[b_{2}(s, x) \eta(s, x) \varphi(x) \mathrm{d} x \mathrm{~d} s+\zeta(s, x) \varphi(x) \mathrm{d} x \mathrm{~d} B(s)\right], \quad \mathcal{P} \text {-a.s. }
\end{aligned}
$$

It is easy to show the following well-posedness result for (6.3) (and hence we omit the proof).

Lemma 6.3. For any $\left(\alpha_{0}, \beta_{0}\right) \in L_{\mathcal{F}_{T}}^{2}\left(\Omega ; H_{0}^{1}(G)\right) \times L_{\mathcal{F}_{T}}^{2}\left(\Omega ; L^{2}(G)\right)$, there is a unique solution $(\alpha, \beta, \eta, \zeta) \in \mathbb{H}_{T}$ to the system (6.3).

Furthermore, we have the following energy estimate.

Proposition 6.4. There is a constant $C>0$, depending only on $T, G$ and $b^{i j}(1 \leq i, j \leq n)$, such that for any solution $(\alpha, \beta, \eta, \zeta)$ to (6.3), and for all $s, t$ satisfying $0 \leq s \leq t \leq \widetilde{T}$, it holds that

$$
\mathbb{E} \int_{G}\left(|\beta(s, x)|^{2}+|\nabla \alpha(s, x)|^{2}\right) \mathrm{d} x \leq C \mathrm{e}^{C\left(r_{1}^{\frac{1}{2-n / p}}+r_{2}^{2}\right)} \widetilde{T} \mathbb{E} \int_{G}\left(|\beta(t, x)|^{2}+|\nabla \alpha(t, x)|^{2}\right) \mathrm{d} x,
$$

and

$$
\mathbb{E} \int_{0}^{\widetilde{T}} \int_{G}\left(|\zeta|^{2}+|\nabla \eta|^{2}\right) \mathrm{d} x \mathrm{~d} t \leq C \mathrm{e}^{C\left(r_{1}^{\frac{1}{2-n / p}}+r_{2}^{2}\right) \widetilde{T}}\left|\left(\alpha_{0}, \beta_{0}\right)\right|_{L_{\mathcal{F}_{\tilde{T}}}^{2}\left(\Omega ; H_{0}^{1}(G)\right) \times L_{\mathcal{F}_{\widetilde{T}}}^{2}\left(\Omega ; L^{2}(G)\right)}^{2} .
$$

Proof of Proposition 6.4. Define a (modified) energy of the system (6.3) as follows:

$$
\widetilde{\mathcal{E}}(t)=\frac{1}{2} \mathbb{E} \int_{G}\left(|\beta(t, x)|^{2}+\sum_{i, j=1}^{n} b^{i j} \alpha_{x_{i}}(t, x) \alpha_{x_{j}}(t, x)+r_{1}^{\frac{2}{2-n / p}}|\alpha(t, x)|^{2}\right) \mathrm{d} x, \quad t \in[0, T] .
$$


Then, by Itô's formula, we get that

$$
\begin{aligned}
\widetilde{\mathcal{E}}(t)-\widetilde{\mathcal{E}}(s)= & \mathbb{E} \int_{s}^{t} \int_{G}\left(b_{1} \alpha \beta+b_{2} \beta \eta\right) \mathrm{d} x \mathrm{~d} \tau+\frac{1}{2} \mathbb{E} \int_{s}^{t} \int_{G}\left(\sum_{i, j=1}^{n} b^{i j} \eta_{x_{i}} \eta_{x_{j}}+\zeta^{2}\right) \mathrm{d} x \mathrm{~d} \tau \\
& +r_{1}^{\frac{2}{2-n / p}} \mathbb{E} \int_{s}^{t} \int_{G} \alpha \beta \mathrm{d} x \mathrm{~d} \tau+\frac{1}{2} r_{1}^{\frac{2}{2-n / p}} \mathbb{E} \int_{s}^{t} \int_{G}|\eta|^{2} \mathrm{~d} x \mathrm{~d} \tau .
\end{aligned}
$$

Set $p_{1}=\frac{2 p}{n-2}$ and $p_{2}=\frac{2 p}{p-n}$. By $\frac{1}{p}+\frac{1}{p_{1}}+\frac{1}{p_{2}}+\frac{1}{2}=1$ and $\frac{1}{2(n / p)^{-1}}+\frac{1}{2(1-n / p)^{-1}}+\frac{1}{2}=1$, and using Hölder's inequality and Sobolev's embedding theorem, we obtain that

$$
\begin{aligned}
\left|\mathbb{E} \int_{G} b_{1}(\tau, x) \alpha(\tau, x) \beta(\tau, x) \mathrm{d} x\right| & \leq \mathbb{E} \int_{G}\left|b_{1}(\tau, x)\right||\alpha(\tau, x)|^{\frac{n}{p}}|\alpha(\tau, x)|^{1-\frac{n}{p}}|\beta(\tau, x)| \mathrm{d} x \\
& \leq r_{1} \mathbb{E}\left(\left.\left.\left.\left.|| \alpha(\tau, \cdot)\right|^{\frac{n}{p}}\right|_{L^{p_{1}(G)}}|| \alpha(\tau, \cdot)\right|^{1-\frac{n}{p}}\right|_{L^{p_{2}(G)}}|\beta(\tau, \cdot)|_{L^{2}(G)}\right) \\
& =r_{1} \mathbb{E}\left(|\alpha(\tau, \cdot)|_{L^{\frac{n}{n-2}}(G)}^{\frac{n}{n-2}}|\alpha(\tau, \cdot)|_{L^{2}(G)}^{1-\frac{n}{p}}|\beta(\tau, \cdot)|_{L^{2}(G)}\right) \\
& =r_{1}^{\frac{1}{2-n / p}} \mathbb{E}\left(|\alpha(\tau, \cdot)|_{L^{\frac{2 n}{n-2}}(G)}^{\frac{1-n / p}{2-n / p}}|\alpha(\tau, \cdot)|_{L^{2}(G)}^{1-\frac{n}{p}}|\beta(\tau, \cdot)|_{L^{2}(G)}\right) .
\end{aligned}
$$

Notice that

$$
\left\{\begin{array}{l}
|\alpha(\tau, \cdot)|_{L^{\frac{n}{n-2}}(G)}^{\frac{n}{p}} \leq C\left[\int_{G}\left(|\beta(\tau, x)|^{2}+\sum_{i, j=1}^{n} b^{i j} \alpha_{x_{i}}(\tau, x) \alpha_{x_{j}}(\tau, x)+r_{1}^{\frac{2}{2-n / p}}|\alpha(\tau, x)|^{2}\right) \mathrm{d} x\right]^{\frac{n}{2 p}}, \\
r_{1}^{\frac{1-n / p}{2-n / p}}|\alpha(\tau, \cdot)|_{L^{2}(G)}^{1-\frac{n}{p}} \leq\left[\int_{G}\left(|\beta(\tau, x)|^{2}+\sum_{i, j=1}^{n} b^{i j} \alpha_{x_{i}}(\tau, x) \alpha_{x_{j}}(\tau, x)+r_{1}^{\frac{2}{2-n / p}}|\alpha(\tau, x)|^{2}\right) \mathrm{d} x\right]^{\frac{1}{2}-\frac{n}{2 p}}, \\
|\beta(\tau, \cdot)|_{L^{2}(G)} \leq\left[\int_{G}\left(|\beta(\tau, x)|^{2}+\sum_{i, j=1}^{n} b^{i j} \alpha_{x_{i}}(\tau, x) \alpha_{x_{j}}(\tau, x)+r_{1}^{\frac{2}{2-n / p}}|\alpha(\tau, x)|^{2}\right) \mathrm{d} x\right]^{\frac{1}{2}} .
\end{array}\right.
$$

We have

$$
\left|\mathbb{E} \int_{G} b_{1}(\tau, x) \alpha(\tau, x) \beta(\tau, x) \mathrm{d} x\right| \leq C r_{1}^{\frac{1}{2-n / p}} \widetilde{\mathcal{E}}(\tau)
$$

By a similar argument, we can also obtain that

$$
r_{1}^{\frac{2}{2-n / p}}\left|\mathbb{E} \int_{G} \alpha(\tau, x) \beta(\tau, x) \mathrm{d} x\right| \leq \frac{1}{2} r_{1}^{\frac{1}{2-n / p}} \mathbb{E} \int_{G}\left[r_{1}^{\frac{2}{2-n / p}} \alpha^{2}(\tau, x)+\beta^{2}(\tau, x)\right] \mathrm{d} x \leq r_{1}^{\frac{1}{2-n / p}} \widetilde{\mathcal{E}}(\tau) .
$$

Further, for a sufficiently small $\epsilon>0$,

$$
\left|\mathbb{E} \int_{s}^{t} \int_{G} b_{2}(\tau, x) \beta(\tau, x) \eta(\tau, x) \mathrm{d} x \mathrm{~d} \tau\right| \leq C(\epsilon) r_{2}^{2} \int_{s}^{t} \widetilde{\mathcal{E}}(\tau) \mathrm{d} \tau+\epsilon \mathbb{E} \int_{s}^{t} \int_{G}|\nabla \eta(\tau, x)|^{2} \mathrm{~d} x \mathrm{~d} \tau .
$$

By (6.9)-(6.12), we find that

$$
\frac{1}{2} \mathbb{E} \int_{s}^{t} \int_{G}\left(|\zeta|^{2}+r_{1}^{\frac{2}{2-n / p}}|\eta|^{2}+\frac{1}{2} \sum_{i, j=1}^{n} b^{i j} \eta_{x_{i}} \eta_{x_{j}}\right) \mathrm{d} x \mathrm{~d} \tau+\widetilde{\mathcal{E}}(s) \leq \widetilde{\mathcal{E}}(t)+C\left(r_{1}^{\frac{1}{2-n / p}}+r_{2}^{2}\right) \int_{s}^{t} \widetilde{\mathcal{E}}(\tau) \mathrm{d} \tau .
$$

This, together with Gronwall's inequality, implies that

$$
\widetilde{\mathcal{E}}(s) \leq \mathrm{e}^{C\left(r_{1}^{\frac{1}{2-n / p}}+r_{2}^{2}\right) \widetilde{T}} \widetilde{\mathcal{E}}(t),
$$

which implies (6.6) and (6.7). 


\section{Proof of Theorem 1.6}

In this section, we shall prove Theorem 1.6.

Proof of Theorem 1.6. We borrow some ideas from [3]. The whole proof is divided into four steps.

Step 1. Note that the solution $y$ to (1.3) may not be zero at $t=0$ and $t=T$. To apply Theorem 5.1 , we need to choose a suitable cutoff function. Set

$$
T_{j}=\frac{T}{2}-\varepsilon_{j} T, \quad T_{j}^{\prime}=\frac{T}{2}+\varepsilon_{j} T, \quad R_{0}=\min _{x \in \bar{G}} \sqrt{\mathrm{d}(x)} \text { and } R_{1}=\max _{x \in \bar{G}} \sqrt{\mathrm{d}(x)},
$$

where $j=0,1,2$ and $0<\varepsilon_{0}<\varepsilon_{1}<\frac{1}{2}$. By (3.2), (1.14) and (1.15), for any $T>T_{0}$, we have that

$$
\phi(0, x)=\phi(T, x) \leq R_{1}^{2}-\frac{c_{1} T^{2}}{4}<0, \quad \forall x \in G .
$$

Therefore, there exists an $\varepsilon_{1} \in\left(0, \frac{1}{2}\right)$, which is close to $\frac{1}{2}$, such that

$$
\phi(t, x) \leq \frac{R_{1}^{2}}{2}-\frac{c_{1} T^{2}}{8}<0, \quad \forall(t, x) \in\left[\left(0, T_{1}\right) \cup\left(T_{1}^{\prime}, T_{1}\right)\right] \times G .
$$

On the other hand, it follows from (3.2) that

$$
\phi\left(\frac{T}{2}, x\right)=\mathrm{d}(x) \geq R_{0}^{2}, \quad \forall x \in G .
$$

Therefore, there is an $\varepsilon_{0} \in\left(0, \frac{1}{2}\right)$, which is close to 0 , such that

$$
\phi(t, x) \geq \frac{R_{0}^{2}}{2}, \quad \forall(t, x) \in\left(T_{0}, T_{0}^{\prime}\right) \times G .
$$

Furthermore, choose a nonnegative function $\xi \in C_{0}^{\infty}(0, T)$ such that

$$
\xi(t)=1 \quad \text { in }\left(T_{1}, T_{1}^{\prime}\right) .
$$

Step 2. In this step, we prove that there is a $\lambda_{1}>0$, such that for any $\lambda \geq \lambda_{1}$,

$$
\lambda \mathbb{E} \int_{Q} \mathrm{e}^{2 \lambda \phi} y^{2} \mathrm{~d} x \mathrm{~d} t \leq C\left(\lambda^{2} \mathbb{E} \int_{0}^{T} \int_{G_{0}} \mathrm{e}^{2 \lambda \phi} y^{2} \mathrm{~d} x \mathrm{~d} t+\mathbb{E}|y|_{L^{2}(J \times G)}^{2}+\mathbb{E} \int_{Q} \mathrm{e}^{2 \lambda \phi} f^{2} \mathrm{~d} x \mathrm{~d} t\right),
$$

where $J=\left(0, T_{1}\right) \cup\left(T_{1}^{\prime}, T\right)$.

To this aim, set $\tilde{y}=\xi y$. Then $\tilde{y}$ satisfies the following forward stochastic hyperbolic equation:

$$
\left\{\begin{array}{lr}
\mathrm{d} \tilde{y}_{t}-\sum_{i, j=1}^{n}\left(b^{i j} \tilde{y}_{x_{i}}\right)_{x_{j}} \mathrm{~d} t=\left(b_{1} \tilde{y}+\tilde{f}\right) \mathrm{d} t+\left(b_{2} \tilde{y}+\xi g\right) \mathrm{d} B(t) & \text { in } Q, \\
\tilde{y}=0 & \text { on } \Sigma, \\
\tilde{y}(0)=\tilde{y}(T)=0 & \text { in } G,
\end{array}\right.
$$

with $\tilde{f}=\xi f+\xi_{t t} y+2 \xi_{t} y_{t}$. By Theorem 5.1, for any $\lambda \geq \lambda_{0}$, we have that

$$
\lambda \mathbb{E} \int_{Q} \mathrm{e}^{2 \lambda \phi} \tilde{y}^{2} \mathrm{~d} x \mathrm{~d} t \leq C\left(\mathbb{E}\left|\mathrm{e}^{\lambda \phi} \tilde{f}\right|_{H^{-1}(Q)}^{2}+\left|\mathrm{e}^{\lambda \phi} b_{1} \tilde{y}\right|_{L_{\mathbb{F}}^{2}\left(0, T ; H^{-1}(G)\right)}^{2}+\lambda^{2} \mathbb{E} \int_{0}^{T} \int_{G_{0}} \mathrm{e}^{2 \lambda \phi} \tilde{y}^{2} \mathrm{~d} x \mathrm{~d} t\right) .
$$


By the definition of $\tilde{f}$, we find that

$$
\begin{aligned}
\mathbb{E}\left|\mathrm{e}^{\lambda \phi} \tilde{f}\right|_{H^{-1}(Q)}^{2} & =\mathbb{E}\left|\mathrm{e}^{\lambda \phi}\left(\xi f+\xi_{t t} y+2 \xi_{t} y_{t}\right)\right|_{H^{-1}(Q)}^{2} \\
& =\sup _{|h|_{L^{2}\left(\Omega ; H_{0}^{1}(Q)\right)}=1}\left|\mathbb{E}\left\langle\mathrm{e}^{\lambda \phi}\left(\xi f+\xi_{t t} y+2 \xi_{t} y_{t}\right), h\right\rangle_{H^{-1}(Q), H_{0}^{1}(Q)}\right|^{2} \\
& \leq\left|\mathrm{e}^{\lambda \phi} f\right|_{L_{\mathbb{F}}^{2}\left(0, T ; L^{2}(G)\right)}^{2}+C \lambda^{2}\left|\mathrm{e}^{\lambda \phi} y\right|_{L_{\mathbb{F}}^{2}\left(J ; L^{2}(G)\right)}^{2} \\
& \leq\left|\mathrm{e}^{\lambda \phi} f\right|_{L_{\mathbb{F}}^{2}\left(0, T ; L^{2}(G)\right)}^{2}+C \lambda^{2} \mathrm{e}^{\lambda\left(R_{1}^{2}-\frac{c_{1} T^{2}}{4}\right)}|y|_{L_{\mathbb{F}}^{2}\left(J ; L^{2}(G)\right)}^{2} .
\end{aligned}
$$

Further, recalling the definition of $r_{1}$ and noting the embedding $L^{2 p / p+2}(G) \hookrightarrow H^{-1}(G)$, we get that

$$
\left|\mathrm{e}^{\lambda \phi} b_{1} \tilde{y}\right|_{L_{\mathbb{F}}^{2}\left(0, T ; H^{-1}(G)\right)} \leq C\left|\mathrm{e}^{\lambda \phi} b_{1} \xi y\right|_{L_{\mathbb{F}}^{2}\left(0, T ; L^{2 p / p+2}(G)\right)} \leq C r_{1}\left|\mathrm{e}^{\lambda \phi} \xi y\right|_{L_{\mathbb{F}}^{2}\left(0, T ; L^{2}(G)\right)} .
$$

Further, by (7.3) and (7.5),

$$
\begin{aligned}
\left|\mathrm{e}^{\lambda \phi} \xi y\right|_{L_{\mathbb{F}}^{2}\left(0, T ; L^{2}(G)\right)}^{2} & =\left|\mathrm{e}^{\lambda \phi} y\right|_{L_{\mathbb{F}}^{2}\left(0, T ; L^{2}(G)\right)}^{2}-\mathbb{E} \int_{Q} \mathrm{e}^{2 \lambda \phi}\left(1-\xi^{2}\right) y^{2} \mathrm{~d} x \mathrm{~d} t \\
& \geq\left|\mathrm{e}^{\lambda \phi} y\right|_{L_{\mathbb{F}}^{2}\left(0, T ; L^{2}(G)\right)}^{2}-C \mathrm{e}^{\left(R_{1}^{2}-c_{1} T^{2} / 4\right) \lambda}|y|_{L_{\mathbb{P}}^{2}\left(J ; L^{2}(G)\right)}^{2} .
\end{aligned}
$$

Therefore, by (7.8)-(7.11), there is a constant $C_{1}=C_{1}(T, G)$, independent of $\lambda$ and $r_{1}$, such that

$$
\begin{aligned}
\left|\mathrm{e}^{\lambda \phi} y\right|_{L_{\mathbb{F}}^{2}\left(0, T ; L^{2}(G)\right) \leq C_{1}}^{2} & \frac{r_{1}^{2}}{\lambda}\left|\mathrm{e}^{\lambda \phi} y\right|_{L_{\mathbb{F}}^{2}\left(0, T ; L^{2}(G)\right)}^{2}+\lambda \mathbb{E} \int_{0}^{T} \int_{G_{0}} \mathrm{e}^{2 \lambda \phi} y^{2} \mathrm{~d} x \mathrm{~d} t \\
& \left.+\mathrm{e}^{\left(R_{1}^{2}-c T^{2} / 4\right) \lambda}(1+\lambda) \mathbb{E}|y|_{L^{2}(J \times G)}^{2}+\frac{1}{\lambda}\left|\mathrm{e}^{\lambda \phi} f\right|_{L_{\mathbb{F}}^{2}\left(0, T ; L^{2}(G)\right)}^{2}\right] .
\end{aligned}
$$

Since $R_{1}^{2}-c T^{2} / 4<0$, one may find a sufficiently large $\lambda_{1}>0$, such that for any $\lambda>\lambda_{1},(7.6)$ holds.

Step 3. We establish an energy estimate for solutions to (1.3). Set

$$
\mathcal{E}(t) \triangleq \frac{1}{2}\left(\mathbb{E}|y(t, \cdot)|_{L^{2}(G)}^{2}+\mathbb{E}\left|y_{t}(t, \cdot)\right|_{H^{-1}(G)}^{2}\right) .
$$

Then by the classical energy estimate, for any $S_{0} \in\left(T_{0}, \frac{T}{2}\right)$ and $S_{0}^{\prime} \in\left(\frac{T}{2}, T_{0}^{\prime}\right)$,

$$
\int_{S_{0}}^{S_{0}^{\prime}} \mathcal{E}(t) \mathrm{d} t \leq C\left(1+r_{1}+r_{2}\right) \mathbb{E} \int_{S_{0}}^{S_{0}^{\prime}} \int_{G} y^{2} \mathrm{~d} x \mathrm{~d} t+C \mathbb{E} \int_{0}^{T} \int_{G}\left(f^{2}+g^{2}\right) \mathrm{d} x \mathrm{~d} t .
$$

On the other hand, we claim that there exists a constant $C>0$, such that

$$
\mathcal{E}(t) \leq C \mathrm{e}^{C\left(r_{1}^{\frac{1}{2-n / p}}+r_{2}^{2}\right)}\left(\mathcal{E}(s)+|(f, g)|_{\left(L_{\mathbb{F}}^{2}\left(0, T ; L^{2}(G)\right)\right)^{2}}^{2}\right), \quad \forall t, s \in[0, T] .
$$

In the following, we only prove the case of $t \geq s$. The other case can be also proved by a similar technique and Proposition 6.1. By Itô's formula, let $\widetilde{T}=t$ in $(6.3)$ and $T=t$ in (1.3). Then it follows that

$$
\begin{aligned}
& \mathbb{E}\left\langle y(t), \beta_{0}\right\rangle_{L^{2}(G)}+\mathbb{E}\left\langle y_{t}(t),-\alpha_{0}\right\rangle_{H^{-1}(G), H_{0}^{1}(G)} \\
& =\mathbb{E}\langle y(s), \beta(s)\rangle_{L^{2}(G)}+\mathbb{E}\left\langle y_{t}(s),-\alpha(s)\right\rangle_{H^{-1}(G), H_{0}^{1}(G)}-\mathbb{E} \int_{s}^{t} \int_{G}(\alpha f+\eta g) \mathrm{d} x \mathrm{~d} t .
\end{aligned}
$$


Denote by $\mathbb{S}$ the unit sphere of the space $L_{\mathcal{F}_{t}}^{2}\left(\Omega ; H_{0}^{1}(G)\right) \times L_{\mathcal{F}_{t}}^{2}\left(\Omega ; L^{2}(G)\right)$. By $(7.16),(6.6)$ and (6.7), we obtain that

$$
\begin{aligned}
\sqrt{2 \mathcal{E}(t)}= & \sup _{\left(\alpha_{0}, \beta_{0}\right) \in \mathbb{S}}\left|\mathbb{E}\left(\left\langle y(t), \beta_{0}\right\rangle_{L^{2}(G)}+\left\langle y_{t}(t),-\alpha_{0}\right\rangle_{H^{-1}(G), H_{0}^{1}(G)}\right)\right| \\
= & \sup _{\left(\alpha_{0}, \beta_{0}\right) \in \mathbb{S}}\left|\mathbb{E}\left[\langle y(s), \beta(s)\rangle_{L^{2}(G)}+\left\langle y_{t}(s),-\alpha(s)\right\rangle_{H^{-1}(G), H_{0}^{1}(G)}-\int_{s}^{t} \int_{G}(\alpha f+\eta g) \mathrm{d} x \mathrm{~d} t\right]\right| \\
\leq & C \sqrt{\mathcal{E}(s)} \sup _{\left(\alpha_{0}, \beta_{0}\right) \in \mathbb{S}}|(\alpha(s), \beta(s))|_{L_{\mathcal{F}_{s}}^{2}\left(\Omega ; H_{0}^{1}(G)\right) \times L_{\mathcal{F}_{\mathcal{S}}}^{2}\left(\Omega ; L^{2}(G)\right)} \\
& +\sup _{\left(\alpha_{0}, \beta_{0}\right) \in \mathbb{S}}|(\alpha, \eta)|_{\left(L_{\mathbb{F}}^{2}\left(s, t ; L^{2}(G)\right)\right)^{2}}|(f, g)|_{\left(L_{\mathbb{F}}^{2}\left(s, t ; L^{2}(G)\right)\right)^{2}} \\
\leq & \left.C \mathrm{e}^{C\left(r_{1}^{2-n / p}\right.}+r_{2}^{2}\right)\left[\sqrt{\mathcal{E}(s)}+|(f, g)|_{\left(L_{\mathbb{F}}^{2}\left(s, t ; L^{2}(G)\right)\right)^{2}}\right] .
\end{aligned}
$$

This implies our claim (7.15).

Step 4. First, it follows from (7.4) that

$$
\mathbb{E} \int_{Q} \mathrm{e}^{2 \lambda \phi} y^{2} \mathrm{~d} x \mathrm{~d} t \geq \mathrm{e}^{R_{0}^{2} \lambda} \mathbb{E} \int_{T_{0}}^{T_{0}^{\prime}} \int_{G} y^{2} \mathrm{~d} x \mathrm{~d} t
$$

Also, (7.15) implies that

$$
\mathbb{E}|y|_{L^{2}(J \times G)}^{2} \leq C \mathrm{e}^{C\left(r_{1}^{\frac{1}{2-n / p}}+r_{2}^{2}\right)}\left(\mathcal{E}(0)+|(f, g)|_{\left(L_{\mathbb{P}}^{2}\left(0, T ; L^{2}(G)\right)\right)^{2}}^{2}\right)
$$

and

$$
C \mathrm{e}^{-C\left(r_{1}^{\frac{1}{2-n / p}}+r_{2}^{2}\right)} \mathcal{E}(0) \leq \int_{S_{0}}^{S_{0}^{\prime}} \mathcal{E}(t) \mathrm{d} t+|(f, g)|_{\left(L_{\mathbb{F}}^{2}\left(0, T ; L^{2}(G)\right)\right)^{2}}^{2}
$$

By (7.17), (7.14) and (7.19), we get that

$$
\mathcal{E}(0) \leq C \mathrm{e}^{C\left(r_{1}^{\frac{1}{2-n / p}}+r_{2}^{2}\right)}\left(\mathrm{e}^{-R_{0}^{2} \lambda} \mathbb{E} \int_{Q} \mathrm{e}^{2 \lambda \phi} y^{2} \mathrm{~d} x \mathrm{~d} t+|(f, g)|_{\left(L_{\mathbb{F}}^{2}\left(0, T ; L^{2}(G)\right)\right)^{2}}^{2}\right) .
$$

Combining the above estimate with (7.6) and (7.18), we find that

$$
\begin{aligned}
\mathcal{E}(0) & \leq C \mathrm{e}^{C\left(r_{1}^{\frac{1}{2-n / p}}+r_{2}^{2}\right)}\left[\mathrm{e}^{-R_{0}^{2} \lambda}\left(\lambda \mathbb{E} \int_{0}^{T} \int_{G_{0}} \mathrm{e}^{2 \lambda \phi} y^{2} \mathrm{~d} x \mathrm{~d} t+\frac{1}{\lambda} \mathbb{E}|y|_{L^{2}(J \times G)}^{2}\right)+|(f, g)|_{\left(L_{\mathbb{F}}^{2}\left(0, T ; L^{2}(G)\right)\right)^{2}}^{2}\right] \\
& \leq C \mathrm{e}^{C\left(r_{1}^{\frac{1}{2-n / p}}+r_{2}^{2}\right)}\left[\mathrm{e}^{-R_{0}^{2} \lambda}\left(\lambda \mathbb{E} \int_{0}^{T} \int_{G_{0}} \mathrm{e}^{2 \lambda \phi} y^{2} \mathrm{~d} x \mathrm{~d} t+\frac{1}{\lambda} \mathcal{E}(0)\right)+|(f, g)|_{\left(L_{\mathbb{F}}^{2}\left(0, T ; L^{2}(G)\right)\right)^{2}}^{2}\right] .
\end{aligned}
$$

Therefore, there exists a sufficiently large constant $\lambda_{3}>0$, such that for any $\lambda>\lambda_{3}$, the desired observability inequality (1.7) holds.

\section{Appendix A. Proof of Proposition 4.1}

In this section, we give a proof of Proposition 4.1. To this aim, we need the following known result.

Lemma A.1 ([4], Prop. 3.5). For any $h>0, m=3,4, \ldots$, and $q_{m}^{j}, w_{m}^{j} \in \mathbb{C}(j=0,1, \ldots, m)$ satisfying $q_{m}^{0}=q_{m}^{m}=0$, we have that

$$
-\sum_{j=1}^{m-1} q_{m}^{j} \frac{w_{m}^{j+1}-2 w_{m}^{j}+w_{m}^{j-1}}{h^{2}}=\sum_{j=0}^{m-1} \frac{q_{m}^{j+1}-q_{m}^{j}}{h} \frac{w_{m}^{j+1}-w_{m}^{j}}{h}=\sum_{j=1}^{m} \frac{q_{m}^{j}-q_{m}^{j-1}}{h} \frac{w_{m}^{j}-w_{m}^{j-1}}{h} .
$$


Proof of Proposition 4.1. The whole proof is divided into four steps.

Step 1. Let $\left\{\left\{\left(z_{m}^{j, k}, r_{1 m}^{j, k}, r_{2 m}^{j, k}, r_{m}^{j, k}\right)\right\}_{j=0}^{m}\right\}_{k=1}^{\infty} \subset \mathcal{A}_{a d}$ be a minimizing sequence of $J(\cdot)$. Thanks to the coercivity of the cost functional, since $z_{m}^{j, k}$ solves an elliptic equation, it can be shown that $\left\{\left\{\left(z_{m}^{j, k}, r_{1 m}^{j, k}, r_{2 m}^{j, k}, r_{m}^{j, k}\right)\right\}_{j=0}^{m}\right\}_{k=1}^{\infty}$ is bounded in $\mathcal{A}_{a d}$. Therefore, there exists a subsequence of $\left\{\left\{\left(z_{m}^{j, k}, r_{1 m}^{j, k}, r_{2 m}^{j, k}, r_{m}^{j, k}\right)\right\}_{j=0}^{m}\right\}_{k=1}^{\infty}$ converging weakly to some $\left\{\left(\hat{z}_{m}^{j}, \hat{r}_{1 m}^{j}, \hat{r}_{2 m}^{j}, \hat{r}_{m}^{j}\right)\right\}_{j=0}^{m} \in \mathcal{A}_{a d}$ in $\left(L_{\mathcal{F}_{j h}}^{2}\left(\Omega ; H_{0}^{1}(G)\right) \times\left(L_{\mathcal{F}_{j h}}^{2}\left(\Omega ; L^{2}(G)\right)\right)^{3}\right)^{m+1}$. Since the functional $J$ is strictly convex, this element is the unique solution to (4.5). By (4.6) and the definition of $\mathcal{A}_{a d}$, it is obvious that $\hat{z}_{m}^{0}=\hat{z}_{m}^{m}=p_{m}^{0}=p_{m}^{m}=0$ in $G$.

Step 2. Fix $\delta_{0 m}^{j} \in L_{\mathcal{F}_{j h}}^{2}\left(\Omega ; H^{2}(G) \cap H_{0}^{1}(G)\right), \delta_{1 m}^{j} \in L_{\mathcal{F}_{j h}}^{2}\left(\Omega ; L^{2}(G)\right)$, and $\delta_{2 m}^{j} \in L_{\mathcal{F}_{j h}}^{2}\left(\Omega ; L^{2}(G)\right)(j=$ $0,1,2, \ldots, m)$ with $\delta_{0 m}^{0}=\delta_{0 m}^{m}=\delta_{2 m}^{0}=\delta_{2 m}^{m} \equiv 0$ and $\delta_{1 m}^{0}=\delta_{1 m}^{1}$. For $\left(\mu_{0}, \mu_{1}, \mu_{2}\right) \in \mathbb{R}^{3}$, put

$$
\left\{\begin{aligned}
r_{m}^{j} \triangleq & \mathbb{E}\left(\frac{\hat{z}_{m}^{j+1}-2 \hat{z}_{m}^{j}+\hat{z}_{m}^{j-1}}{h^{2}} \mid \mathcal{F}_{j h}\right)+\mathbb{E}\left(\frac{\delta_{0 m}^{j+1}-2 \delta_{0 m}^{j}+\delta_{0 m}^{j-1}}{h^{2}} \mid \mathcal{F}_{j h}\right) \mu_{0} \\
& -\sum_{j_{1}, j_{2}=1}^{n} \partial_{x_{j_{2}}}\left(b^{j_{1} j_{2}} \partial_{x_{j_{1}}}\left(\hat{z}_{m}^{j}+\mu_{0} \delta_{0 m}^{j}\right)\right)-\mathbb{E}\left(\frac{\hat{r}_{1 m}^{j+1}-\hat{r}_{1 m}^{j}}{h} \mid \mathcal{F}_{j h}\right) \\
& -\mathbb{E}\left(\frac{\delta_{1 m}^{j+1}-\delta_{1 m}^{j}}{h} \mid \mathcal{F}_{j h}\right) \mu_{1}-\hat{r}_{2 m}^{j}-\mu_{2} \delta_{2 m}^{j}-\lambda y_{m}^{j} \mathrm{e}^{2 \lambda \phi_{m}^{j}}, \quad 1 \leq j \leq m-1 ; \\
r_{m}^{0}= & r_{m}^{m}=0 .
\end{aligned}\right.
$$

Then $\left\{\left(\hat{z}_{m}^{j}+\mu_{0} \delta_{0 m}^{j}, \hat{r}_{1 m}^{j}+\mu_{1} \delta_{1 m}^{j}, \hat{r}_{2 m}^{j}+\mu_{2} \delta_{2 m}^{j}, r_{m}^{j}\right)\right\}_{j=0}^{m} \in \mathcal{A}_{a d}$. Define a function $g(\cdot, \cdot, \cdot)$ in $\mathbb{R}^{3}$ by

$$
g\left(\mu_{0}, \mu_{1}, \mu_{2}\right)=J\left(\left\{\left(\hat{z}_{m}^{j}+\mu_{0} \delta_{0 m}^{j}, \hat{r}_{1 m}^{j}+\mu_{1} \delta_{1 m}^{j}, \hat{r}_{2 m}^{j}+\mu_{2} \delta_{2 m}^{j}, r_{m}^{j}\right)\right\}_{j=0}^{m}\right) .
$$

Since $g$ has a minimum at $(0,0,0)$, we get that $\nabla g(0,0,0)=0$.

By $\frac{\partial g(0,0,0)}{\partial \mu_{1}}=\frac{\partial g(0,0,0)}{\partial \mu_{2}}=0$, noting that $\left\{\left(\hat{z}_{m}^{j}, \hat{r}_{1 m}^{j}, \hat{r}_{2 m}^{j}, \hat{r}_{m}^{j}\right)\right\}_{j=0}^{m}$ satisfy the first equation of (4.3), we find that

$$
\begin{aligned}
& -K \mathbb{E} \sum_{j=1}^{m-1} \int_{G} \hat{r}_{m}^{j} \mathbb{E}\left(\frac{\delta_{1 m}^{j+1}-\delta_{1 m}^{j}}{h} \mid \mathcal{F}_{j h}\right) \mathrm{d} x+\mathbb{E} \sum_{j=1}^{m} \int_{G} \varrho \frac{\hat{r}_{1 m}^{j} \delta_{1 m}^{j}}{\lambda^{2}} \mathrm{e}^{-2 \lambda \phi_{m}^{j}} \mathrm{~d} x \\
& =K \mathbb{E} \sum_{j=1}^{m} \int_{G} \frac{\hat{r}_{m}^{j}-\hat{r}_{m}^{j-1}}{h} \delta_{1 m}^{j} \mathrm{~d} x+\mathbb{E} \sum_{j=1}^{m} \int_{G} \varrho \frac{\hat{r}_{1 m}^{j} \delta_{1 m}^{j}}{\lambda^{2}} \mathrm{e}^{-2 \lambda \phi_{m}^{j}} \mathrm{~d} x \\
& =K \mathbb{E} \sum_{j=1}^{m} \int_{G}\left(\frac{\hat{r}_{m}^{j}-\hat{r}_{m}^{j-1}}{h}+\varrho \frac{\hat{r}_{1 m}^{j}}{\lambda^{2}} \mathrm{e}^{-2 \lambda \phi_{m}^{j}}\right) \delta_{1 m}^{j} \mathrm{~d} x=0,
\end{aligned}
$$

and

$$
-K \mathbb{E} \sum_{j=1}^{m-1} \int_{G} \hat{r}_{m}^{j} \delta_{2 m}^{j} \mathrm{~d} x+\mathbb{E} \sum_{j=1}^{m-1} \int_{G} \varrho \frac{\hat{r}_{2 m}^{j} \delta_{2 m}^{j}}{\lambda^{4}} \mathrm{e}^{-2 \lambda \phi_{m}^{j}} \mathrm{~d} x=-\mathbb{E} \sum_{j=1}^{m-1} \int_{G}\left(K \hat{r}_{m}^{j}-\varrho \frac{\hat{r}_{2 m}^{j}}{\lambda^{4}} \mathrm{e}^{-2 \lambda \phi_{m}^{j}}\right) \delta_{2 m}^{j} \mathrm{~d} x=0,
$$

which, combined with (4.6), gives (4.8).

On the other hand, by $\frac{\partial g(0,0,0)}{\partial \mu_{0}}=0$, we have that

$$
\mathbb{E} \sum_{j=1}^{m-1} \int_{G}\left\{K \hat{r}_{m}^{j}\left[\mathbb{E}\left(\frac{\delta_{0 m}^{j+1}-2 \delta_{0 m}^{j}+\delta_{0 m}^{j-1}}{h^{2}} \mid \mathcal{F}_{j h}\right)-\sum_{j_{1}, j_{2}=1}^{n} \partial_{x_{j_{2}}}\left(b^{j_{1} j_{2}} \partial_{x_{j_{1}}} \delta_{0 m}^{j}\right)\right]+\hat{z}_{m}^{j} \delta_{0 m}^{j} \mathrm{e}^{-2 \lambda \phi_{m}^{j}}\right\} \mathrm{d} x=0,
$$


which, combined with $p_{m}^{0}=p_{m}^{m}=\delta_{0 m}^{0}=\delta_{0 m}^{m}=0$ in $G$, implies that (4.9) holds. By means of the regularity theory for elliptic equations of second order, one finds that $\hat{z}_{m}^{j}, p_{m}^{j} \in L_{\mathcal{F}_{j h}}^{2}\left(\Omega ; H^{2}(G) \cap H_{0}^{1}(G)\right), 1 \leq j \leq m-1$.

Step 3. Recalling that $\left\{\left(\hat{z}_{m}^{j}, \hat{r}_{1 m}^{j}, \hat{r}_{2 m}^{j}, \hat{r}_{m}^{j}\right)\right\}_{j=0}^{m}$ satisfy (4.3), and noting (4.8)-(4.9) and $p_{m}^{j}=K \hat{r}_{m}^{j}$, one gets

$$
\begin{aligned}
0= & \mathbb{E} \sum_{j=1}^{m-1} \int_{G}\left[\mathbb{E}\left(\frac{\hat{z}_{m}^{j+1}-2 \hat{z}_{m}^{j}+\hat{z}_{m}^{j-1}}{h^{2}} \mid \mathcal{F}_{j h}\right)-\sum_{j_{1}, j_{2}=1}^{n} \partial_{x_{j_{2}}}\left(b^{j_{1} j_{2}} \partial_{x_{j_{1}}} \hat{z}_{m}^{j}\right)\right. \\
& \left.-\mathbb{E}\left(\frac{\hat{r}_{1 m}^{j+1}-\hat{r}_{1 m}^{j}}{h} \mid \mathcal{F}_{j h}\right)-\hat{r}_{2 m}^{j}-\lambda y_{m}^{j} \mathrm{e}^{2 \lambda \phi_{m}^{j}}-\hat{r}_{m}^{j}\right] p_{m}^{j} \mathrm{~d} x \\
= & \mathbb{E} \sum_{j=1}^{m-1} \int_{G}\left[\mathbb{E}\left(\frac{p_{m}^{j+1}-2 p_{m}^{j}+p_{m}^{j-1}}{h^{2}} \mid \mathcal{F}_{j h}\right)-\sum_{j_{1}, j_{2}=1}^{n} \partial_{x_{j_{2}}}\left(b^{j_{1} j_{2}} \partial_{x_{j_{1}}} p_{m}^{j}\right)\right] \hat{z}_{m}^{j} \mathrm{~d} x \\
& +\mathbb{E} \sum_{j=1}^{m} \int_{G} \frac{p_{m}^{j}-p_{m}^{j-1}}{h} \hat{r}_{1 m}^{j} \mathrm{~d} x-\mathbb{E} \sum_{j=1}^{m-1} \int_{G}\left(\hat{r}_{2 m}^{j}+\lambda y_{m}^{j} \mathrm{e}^{2 \lambda \phi_{m}^{j}}+\hat{r}_{m}^{j}\right) p_{m}^{j} \mathrm{~d} x \\
= & -\mathbb{E} \sum_{j=1}^{m-1}\left[\int_{G}\left|\hat{z}_{m}^{j}\right|^{2} \mathrm{e}^{-2 \lambda \phi_{m}^{j}} \mathrm{~d} x+\int_{G} \varrho\left(\frac{\left|\hat{r}_{1 m}^{j}\right|^{2}}{\lambda^{2}}+\frac{\left|\hat{r}_{2 m}^{j}\right|^{2}}{\lambda^{4}}\right) \mathrm{e}^{-2 \lambda \phi_{m}^{j}} \mathrm{~d} x+K \int_{G}\left|\hat{r}_{m}^{j}\right|^{2} \mathrm{~d} x\right] \\
& -\mathbb{E} \int_{G} \varrho \frac{\left|\hat{r}_{1 m}^{m}\right|^{2}}{\lambda^{2}} \mathrm{e}^{-2 \lambda \phi_{m}^{m}} \mathrm{~d} x-\lambda \mathbb{E} \sum_{j=1}^{m-1} \int_{G} y_{m}^{j} \mathrm{e}^{2 \lambda \phi_{m}^{j}} p_{m}^{j} \mathrm{~d} x .
\end{aligned}
$$

By (4.8) and (A.3), there is a constant $C=C(K, \lambda)>0$, such that

$$
\begin{gathered}
\mathbb{E} \sum_{j=1}^{m-1}\left[\int_{G}\left|\hat{z}_{m}^{j}\right|^{2} \mathrm{e}^{-2 \lambda \phi_{m}^{j}} \mathrm{~d} x+\int_{G} \varrho\left(\frac{\left|\hat{r}_{1 m}^{j}\right|^{2}}{\lambda^{2}}+\frac{\left|\hat{r}_{2 m}^{j}\right|^{2}}{\lambda^{4}}\right) \mathrm{e}^{-2 \lambda \phi_{m}^{j}} \mathrm{~d} x+K \int_{G}\left|\hat{r}_{m}^{j}\right|^{2} \mathrm{~d} x\right] \\
+\mathbb{E} \int_{G} \varrho \frac{\left|\hat{r}_{1 m}^{m}\right|^{2}}{\lambda^{2}} \mathrm{e}^{-2 \lambda \phi_{m}^{m}} \mathrm{~d} x \leq C \mathbb{E} \sum_{j=1}^{m-1} \int_{G}\left|y_{m}^{j}\right|^{2} \mathrm{e}^{2 \lambda \phi_{m}^{j}} \mathrm{~d} x .
\end{gathered}
$$

This implies (4.10).

Step 4. Noting that (4.9) holds and $p_{m}^{0}=\hat{z}_{m}^{0}=p_{m}^{m}=\hat{z}_{m}^{m}=0$, we obtain that

$$
\left\{\begin{array}{cc}
\frac{\mathbb{E}\left(p_{m}^{3} \mid \mathcal{F}_{h}\right)-4 \mathbb{E}\left(p_{m}^{2} \mid \mathcal{F}_{h}\right)+5 p_{m}^{1}}{h^{4}}-\sum_{j_{1}, j_{2}=1}^{n} \partial_{x_{j_{2}}}\left[b^{j_{1} j_{2}} \partial_{x_{j_{1}}} \mathbb{E}\left(\frac{p_{m}^{2}-2 p_{m}^{1}+p_{m}^{0}}{h^{2}} \mid \mathcal{F}_{h}\right)\right] & \\
+\frac{\mathbb{E}\left(\hat{z}_{m}^{2} \mid \mathcal{F}_{h}\right) \mathrm{e}^{-2 \lambda \phi_{m}^{2}}-2 \hat{z}_{m}^{1} \mathrm{e}^{-2 \lambda \phi_{m}^{1}}+\hat{z}_{m}^{0} \mathrm{e}^{-2 \lambda \phi_{m}^{0}}}{h^{2}}=0 & \text { in } G, \\
\frac{4 p_{m}^{m-1}+\mathbb{E}\left(p_{m}^{m-1} \mid \mathcal{F}_{(m-2) h}\right)-4 p_{m}^{m-2}+p_{m}^{m-3}}{h^{4}}-\sum_{j_{1}, j_{2}=1}^{n} \partial_{x_{j_{2}}}\left[b^{j_{1} j_{2}} \partial_{x_{j_{1}}} \mathbb{E}\left(\frac{p_{m}^{m}-2 p_{m}^{m-1}+p_{m}^{m-2}}{h^{2}} \mid \mathcal{F}_{(m-1) h}\right)\right] & \text { in } G, \\
+\frac{\hat{z}_{m}^{m} \mathrm{e}^{-2 \lambda \phi_{m}^{m}}-2 \hat{z}_{m}^{m-1} \mathrm{e}^{-2 \lambda \phi_{m}^{m-1}}+\hat{z}_{m}^{m-2} \mathrm{e}^{-2 \lambda \phi_{m}^{m-2}}}{h^{2}}=0 & 0
\end{array}\right.
$$

and for $j=2, \ldots, m-2$,

$$
\begin{aligned}
& \frac{\mathbb{E}\left(p_{m}^{j+2} \mid \mathcal{F}_{j h}\right)-4 \mathbb{E}\left(p_{m}^{j+1} \mid \mathcal{F}_{j h}\right)+5 p_{m}^{j}+\mathbb{E}\left(p_{m}^{j} \mid \mathcal{F}_{(j-1) h}\right)-4 p_{m}^{j-1}+p_{m}^{j-2}}{h^{4}} \\
& -\sum_{j_{1}, j_{2}=1}^{n} \partial_{x_{j_{2}}}\left[b^{j_{1} j_{2}} \partial_{x_{j_{1}}} \mathbb{E}\left(\frac{p_{m}^{j+1}-2 p_{m}^{j}+p_{m}^{j-1}}{h^{2}} \mid \mathcal{F}_{j h}\right)\right] \\
& +\frac{\mathbb{E}\left(\hat{z}_{m}^{j+1} \mid \mathcal{F}_{j h}\right) \mathrm{e}^{-2 \lambda \phi_{m}^{j+1}}-2 \hat{z}_{m}^{j} \mathrm{e}^{-2 \lambda \phi_{m}^{j}}+\hat{z}_{m}^{j-1} \mathrm{e}^{-2 \lambda \phi_{m}^{j-1}}}{h^{2}}=0 \quad \text { in } G .
\end{aligned}
$$


By (4.3),

$$
\begin{aligned}
0= & \mathbb{E} \sum_{j=1}^{m-1} \int_{G}\left[\mathbb{E}\left(\frac{\hat{z}_{m}^{j+1}-2 \hat{z}_{m}^{j}+\hat{z}_{m}^{j-1}}{h^{2}} \mid \mathcal{F}_{j h}\right)-\sum_{j_{1}, j_{2}=1}^{n} \partial_{x_{j_{2}}}\left(b^{j_{1} j_{2}} \partial_{x_{j_{1}}} \hat{z}_{m}^{j}\right)\right. \\
& \left.-\mathbb{E}\left(\frac{\hat{r}_{1 m}^{j+1}-\hat{r}_{1 m}^{j}}{h} \mid \mathcal{F}_{j h}\right)-\hat{r}_{2 m}^{j}-\lambda y_{m}^{j} \mathrm{e}^{2 \lambda \phi_{m}^{j}}-\hat{r}_{m}^{j}\right] \mathbb{E}\left(\frac{p_{m}^{j+1}-2 p_{m}^{j}+p_{m}^{j-1}}{h^{2}} \mid \mathcal{F}_{j h}\right) \mathrm{d} x .
\end{aligned}
$$

Using $\hat{z}_{m}^{0}=\hat{z}_{m}^{m}=p_{m}^{0}=p_{m}^{m}=0$ again, we get that

$$
\begin{aligned}
& \mathbb{E} \sum_{j=1}^{m-1} \int_{G} \mathbb{E}\left(\frac{\hat{z}_{m}^{j+1}-2 \hat{z}_{m}^{j}+\hat{z}_{m}^{j-1}}{h^{2}} \mid \mathcal{F}_{j h}\right) \mathbb{E}\left(\frac{p_{m}^{j+1}-2 p_{m}^{j}+p_{m}^{j-1}}{h^{2}} \mid \mathcal{F}_{j h}\right) \mathrm{d} x \\
= & \mathbb{E} \sum_{j=2}^{m-1} \int_{G} \mathbb{E}\left(\hat{z}_{m}^{j} \mid \mathcal{F}_{(j-1) h}\right) \mathbb{E}\left(\frac{p_{m}^{j}-2 p_{m}^{j-1}+p_{m}^{j-2}}{h^{4}} \mid \mathcal{F}_{(j-1) h}\right) \mathrm{d} x \\
& -2 \mathbb{E} \sum_{j=1}^{m-1} \int_{G} \hat{z}_{m}^{j} \mathbb{E}\left(\frac{p_{m}^{j+1}-2 p_{m}^{j}+p_{m}^{j-1}}{h^{2}} \mid \mathcal{F}_{j h}\right) \mathrm{d} x+\mathbb{E} \sum_{j=1}^{m-2} \int_{G} \hat{z}_{m}^{j} \mathbb{E}\left(\frac{p_{m}^{j+2}-2 p_{m}^{j+1}+p_{m}^{j}}{h^{4}} \mid \mathcal{F}_{(j+1) h}\right) \mathrm{d} x \\
= & \mathbb{E} \int_{G}\left[\hat{z}_{m}^{1} \frac{\mathbb{E}\left(p_{m}^{3} \mid \mathcal{F}_{h}\right)-4 \mathbb{E}\left(p_{m}^{2} \mid \mathcal{F}_{h}\right)+5 p_{m}^{1}}{h^{4}}+\hat{z}_{m}^{m-1} \frac{4 p_{m}^{m-1}+\mathbb{E}\left(p_{m}^{m-1} \mid \mathcal{F}_{(m-2) h}\right)-4 p_{m}^{m-2}+p_{m}^{m-3}}{h^{4}}\right] \mathrm{d} x \\
& +\mathbb{E} \sum_{j=2}^{m-2} \int_{G} \frac{\mathbb{E}\left(p_{m}^{j+2} \mid \mathcal{F}_{j h}\right)-4 \mathbb{E}\left(p_{m}^{j+1} \mid \mathcal{F}_{j h}\right)+5 p_{m}^{j}+\mathbb{E}\left(p_{m}^{j} \mid \mathcal{F}_{(j-1) h}\right)-4 p_{m}^{j-1}+p_{m}^{j-2}}{h^{4}} \hat{z}_{m}^{j} \mathrm{~d} x .
\end{aligned}
$$

Therefore,

$$
\begin{array}{r}
\mathbb{E} \sum_{j=1}^{m-1} \int_{G} \mathbb{E}\left(\frac{\hat{z}_{m}^{j+1}-2 \hat{z}_{m}^{j}+\hat{z}_{m}^{j-1}}{h^{2}} \mid \mathcal{F}_{j h}\right) \mathbb{E}\left(\frac{p_{m}^{j+1}-2 p_{m}^{j}+p_{m}^{j-1}}{h^{2}} \mid \mathcal{F}_{j h}\right) \mathrm{d} x \\
=\mathbb{E} \sum_{j=1}^{m-1} \int_{G} \hat{z}_{m}^{j}\left\{\sum_{j_{1}, j_{2}=1}^{n} \partial_{x_{j_{2}}}\left[b^{j_{1} j_{2}} \partial_{x_{j_{1}}} \mathbb{E}\left(\frac{p_{m}^{j+1}-2 p_{m}^{j}+p_{m}^{j-1}}{h^{2}} \mid \mathcal{F}_{j h}\right)\right]\right. \\
\left.-\frac{\mathbb{E}\left(\hat{z}_{m}^{j+1} \mid \mathcal{F}_{j h}\right) \mathrm{e}^{-2 \lambda \phi_{m}^{j+1}}-2 \hat{z}_{m}^{j} \mathrm{e}^{-2 \lambda \phi_{m}^{j}}+\hat{z}_{m}^{j-1} \mathrm{e}^{-2 \lambda \phi_{m}^{j-1}}}{h^{2}}\right\} \mathrm{d} x .
\end{array}
$$

Noting that $\left.z_{m}^{j}\right|_{\Gamma}=\left.p_{m}^{j}\right|_{\Gamma}=0(j=0,1, \ldots, m)$, one has

$$
\begin{aligned}
& \mathbb{E} \sum_{j=1}^{m-1} \int_{G} \sum_{j_{1}, j_{2}=1}^{n} \partial_{x_{j_{2}}}\left(b^{j_{1} j_{2}} \partial_{x_{j_{1}}} \hat{z}_{m}^{j}\right) \mathbb{E}\left(\frac{p_{m}^{j+1}-2 p_{m}^{j}+p_{m}^{j-1}}{h^{2}} \mid \mathcal{F}_{j h}\right) \mathrm{d} x \\
& =\mathbb{E} \sum_{j=1}^{m-1} \int_{G} \hat{z}_{m}^{j} \sum_{j_{1}, j_{2}=1}^{n} \partial_{x_{j_{2}}}\left[b^{j_{1} j_{2}} \partial_{x_{j_{1}}} \mathbb{E}\left(\frac{p_{m}^{j+1}-2 p_{m}^{j}+p_{m}^{j-1}}{h^{2}} \mid \mathcal{F}_{j h}\right)\right] \mathrm{d} x .
\end{aligned}
$$

Then by (A.6), we obtain that

$$
\begin{aligned}
0= & -\mathbb{E} \sum_{j=1}^{m-1} \int_{G}\left[\hat{z}_{m}^{j} \frac{\mathbb{E}\left(\hat{z}_{m}^{j+1} \mid \mathcal{F}_{j h}\right) \mathrm{e}^{-2 \lambda \phi_{m}^{j+1}}-2 \hat{z}_{m}^{j} \mathrm{e}^{-2 \lambda \phi_{m}^{j}}+\hat{z}_{m}^{j-1} \mathrm{e}^{-2 \lambda \phi_{m}^{j-1}}}{h^{2}}\right. \\
& \left.+\left(\frac{\mathbb{E}\left(\hat{r}_{1 m}^{j+1} \mid \mathcal{F}_{j h}\right)-\hat{r}_{1 m}^{j}}{h}+\hat{r}_{2 m}^{j}+\lambda y_{m}^{j} \mathrm{e}^{2 \lambda \phi_{m}^{j}}+\hat{r}_{m}^{j}\right) \mathbb{E}\left(\frac{p_{m}^{j+1}-2 p_{m}^{j}+p_{m}^{j-1}}{h^{2}} \mid \mathcal{F}_{j h}\right)\right] \mathrm{d} x .
\end{aligned}
$$


It follows from Lemma A.1 that

$$
\begin{aligned}
& -\mathbb{E} \sum_{j=1}^{m-1} \int_{G}\left(\hat{z}_{m}^{j} \frac{\mathbb{E}\left(\hat{z}_{m}^{j+1} \mid \mathcal{F}_{j h}\right) \mathrm{e}^{-2 \lambda \phi_{m}^{j+1}}-2 \hat{z}_{m}^{j} \mathrm{e}^{-2 \lambda \phi_{m}^{j}}+\hat{z}_{m}^{j-1} \mathrm{e}^{-2 \lambda \phi_{m}^{j-1}}}{h^{2}}\right) \mathrm{d} x \\
& =\mathbb{E} \sum_{j=0}^{m-1} \int_{G} \frac{\left(\hat{z}_{m}^{j+1}-\hat{z}_{m}^{j}\right)}{h} \frac{\left(\hat{z}_{m}^{j+1} \mathrm{e}^{-2 \lambda \phi_{m}^{j+1}}-\hat{z}_{m}^{j} \mathrm{e}^{-2 \lambda \phi_{m}^{j}}\right)}{h} \mathrm{~d} x \\
& =\mathbb{E} \sum_{j=0}^{m-1} \int_{G}\left[\frac{\left(\hat{z}_{m}^{j+1}-\hat{z}_{m}^{j}\right)^{2}}{h^{2}} \mathrm{e}^{-2 \lambda \phi_{m}^{j}}+\frac{\hat{z}_{m}^{j+1}-\hat{z}_{m}^{j}}{h} \frac{\mathrm{e}^{-2 \lambda \phi_{m}^{j+1}}-\mathrm{e}^{-2 \lambda \phi_{m}^{j}}}{h} \hat{z}_{m}^{j+1}\right] \mathrm{d} x .
\end{aligned}
$$

By Lemma A.1 and $p_{m}^{j}=K \hat{r}_{m}^{j}$, we have that

$$
-\mathbb{E} \sum_{j=1}^{m-1} \int_{G}\left[\hat{r}_{m}^{j} \mathbb{E}\left(\frac{p_{m}^{j+1}-2 p_{m}^{j}+p_{m}^{j-1}}{h^{2}} \mid \mathcal{F}_{j h}\right)\right] \mathrm{d} x=K \mathbb{E} \sum_{j=0}^{m-1} \int_{G} \frac{\left(\hat{r}_{m}^{j+1}-\hat{r}_{m}^{j}\right)^{2}}{h^{2}} \mathrm{~d} x .
$$

Further, by (4.8) and Lemma A.1, we get that

$$
\begin{aligned}
& -\mathbb{E} \sum_{j=1}^{m-1} \int_{G} \mathbb{E}\left(\frac{\hat{r}_{1 m}^{j+1}-\hat{r}_{1 m}^{j}}{h} \mid \mathcal{F}_{j h}\right) \mathbb{E}\left(\frac{p_{m}^{j+1}-2 p_{m}^{j}+p_{m}^{j-1}}{h^{2}} \mid \mathcal{F}_{j h}\right) \mathrm{d} x \\
& =-\mathbb{E} \sum_{j=1}^{m-1} \int_{G} \mathbb{E}\left(\frac{\hat{r}_{1 m}^{j+1}-\hat{r}_{1 m}^{j}}{h} \mid \mathcal{F}_{j h}\right) \frac{1}{h} \mathbb{E}\left(\frac{p_{m}^{j+1}-p_{m}^{j}}{h}-\frac{p_{m}^{j}-p_{m}^{j-1}}{h} \mid \mathcal{F}_{j h}\right) \mathrm{d} x .
\end{aligned}
$$

Therefore,

$$
\begin{aligned}
- & \mathbb{E} \sum_{j=1}^{m-1} \int_{G} \mathbb{E}\left(\frac{\hat{r}_{1 m}^{j+1}-\hat{r}_{1 m}^{j}}{h} \mid \mathcal{F}_{j h}\right) \mathbb{E}\left(\frac{p_{m}^{j+1}-2 p_{m}^{j}+p_{m}^{j-1}}{h^{2}} \mid \mathcal{F}_{j h}\right) \mathrm{d} x \\
= & \mathbb{E} \sum_{j=1}^{m-1} \int_{G} \frac{\varrho}{\lambda^{2}} \mathbb{E}\left(\frac{\hat{r}_{1 m}^{j+1}-\hat{r}_{1 m}^{j}}{h} \mid \mathcal{F}_{j h}\right) \mathbb{E}\left(\frac{\hat{r}_{1 m}^{j+1} \mathrm{e}^{-2 \lambda \phi_{m}^{j+1}}-\hat{r}_{1 m}^{j} \mathrm{e}^{-2 \lambda \phi_{m}^{j}}}{h} \mid \mathcal{F}_{j h}\right) \mathrm{d} x \\
= & \mathbb{E} \sum_{j=1}^{m-1} \int_{G} \frac{\varrho}{\lambda^{2}}\left\{\frac{\left[\mathbb{E}\left(\hat{r}_{1 m}^{j+1}-\hat{r}_{1 m}^{j} \mid \mathcal{F}_{j h}\right)\right]^{2}}{h^{2}} \mathrm{e}^{-2 \lambda \phi_{m}^{j}}\right. \\
& \left.+\mathbb{E}\left(\frac{\hat{r}_{1 m}^{j+1}-\hat{r}_{1 m}^{j}}{h} \mid \mathcal{F}_{j h}\right) \frac{\mathrm{e}^{-2 \lambda \phi_{m}^{j+1}}-\mathrm{e}^{-2 \lambda \phi_{m}^{j}}}{h} \mathbb{E}\left(\hat{r}_{1 m}^{j+1} \mid \mathcal{F}_{j h}\right)\right\} \mathrm{d} x,
\end{aligned}
$$

and

$$
\begin{aligned}
& -\mathbb{E} \sum_{j=1}^{m-1} \int_{G}\left(\lambda y_{m}^{j} \mathrm{e}^{2 \lambda \phi_{m}^{j}}+\hat{r}_{2 m}^{j}\right) \mathbb{E}\left(\frac{p_{m}^{j+1}-2 p_{m}^{j}+p_{m}^{j-1}}{h^{2}} \mid \mathcal{F}_{j h}\right) \mathrm{d} x \\
= & -\mathbb{E} \sum_{j=1}^{m-1} \int_{G} \lambda y_{m}^{j} \mathrm{e}^{2 \lambda \phi_{m}^{j}} \frac{1}{h} \mathbb{E}\left(\frac{p_{m}^{j+1}-p_{m}^{j}}{h}-\frac{p_{m}^{j}-p_{m}^{j-1}}{h} \mid \mathcal{F}_{j h}\right) \mathrm{d} x+\mathbb{E} \sum_{j=0}^{m-1} \int_{G} \frac{\left(\hat{r}_{2 m}^{j+1}-\hat{r}_{2 m}^{j}\right)}{h} \frac{p_{m}^{j+1}-p_{m}^{j}}{h} \mathrm{~d} x \\
= & \lambda \mathbb{E} \sum_{j=1}^{m-1} \int_{G} \frac{\varrho}{\lambda^{2}} y_{m}^{j}\left[\mathbb{E}\left(\frac{\hat{r}_{1 m}^{j+1}-\hat{r}_{1 m}^{j}}{h} \mathrm{e}^{-2 \lambda \phi_{m}^{j}} \mid \mathcal{F}_{j h}\right)+\frac{\left(\mathrm{e}^{-2 \lambda \phi_{m}^{j+1}}-\mathrm{e}^{-2 \lambda \phi_{m}^{j}}\right)}{h} \mathbb{E}\left(\hat{r}_{1 m}^{j+1} \mid \mathcal{F}_{j h}\right)\right] \mathrm{d} x \\
& +\mathbb{E} \sum_{j=0}^{m-1} \int_{G} \frac{\varrho}{\lambda^{4}}\left[\frac{\left(\hat{r}_{2 m}^{j+1}-\hat{r}_{2 m}^{j}\right)^{2}}{h^{2}} \mathrm{e}^{-2 \lambda \phi_{m}^{j}}+\frac{\left(\hat{r}_{2 m}^{j+1}-\hat{r}_{2 m}^{j}\right)}{h} \frac{\left(\mathrm{e}^{-2 \lambda \phi_{m}^{j+1}}-\mathrm{e}^{\left.-2 \lambda \phi_{m}^{j}\right)}\right.}{h} \hat{r}_{2 m}^{j+1}\right] \mathrm{d} x .
\end{aligned}
$$


By (A.8)-(A.11), it follows that

$$
\begin{aligned}
& \mathbb{E} \sum_{j=0}^{m-1} \int_{G}\left\{\frac{\left(\hat{z}_{m}^{j+1}-\hat{z}_{m}^{j}\right)^{2}}{h^{2}} \mathrm{e}^{-2 \lambda \phi_{m}^{j}}+\frac{\varrho}{\lambda^{2}} \frac{\left[\mathbb{E}\left(\hat{r}_{1 m}^{j+1}-\hat{r}_{1 m}^{j} \mid \mathcal{F}_{j h}\right)\right]^{2}}{h^{2}} \mathrm{e}^{-2 \lambda \phi_{m}^{j}}\right. \\
& \left.+\frac{\varrho}{\lambda^{4}} \frac{\left(\hat{r}_{2 m}^{j+1}-\hat{r}_{2 m}^{j}\right)^{2}}{h^{2}} \mathrm{e}^{-2 \lambda \phi_{m}^{j}}+K \frac{\left(\hat{r}_{m}^{j+1}-\hat{r}_{m}^{j}\right)^{2}}{h^{2}}\right\} \mathrm{d} x \\
& =-\mathbb{E} \sum_{j=0}^{m-1} \int_{G} \frac{\left(\hat{z}_{m}^{j+1}-\hat{z}_{m}^{j}\right)}{h} \frac{\left(\mathrm{e}^{-2 \lambda \phi_{m}^{j+1}}-\mathrm{e}^{-2 \lambda \phi_{m}^{j}}\right)}{h} \hat{z}_{m}^{j+1} \mathrm{~d} x \\
& -\mathbb{E} \sum_{j=1}^{m-1} \int_{G} \frac{\varrho}{\lambda^{2}} \mathbb{E}\left(\frac{\hat{r}_{1 m}^{j+1}-\hat{r}_{1 m}^{j}}{h} \mid \mathcal{F}_{j h}\right) \frac{\left(\mathrm{e}^{-2 \lambda \phi_{m}^{j+1}}-\mathrm{e}^{-2 \lambda \phi_{m}^{j}}\right)}{h} \mathbb{E}\left(\hat{r}_{1 m}^{j+1} \mid \mathcal{F}_{j h}\right) \mathrm{d} x \\
& -\lambda \mathbb{E} \sum_{j=1}^{m-1} \int_{G} \frac{\varrho}{\lambda^{2}} y_{m}^{j}\left[\mathbb{E}\left(\frac{\hat{r}_{1 m}^{j+1}-\hat{r}_{1 m}^{j}}{h} \mathrm{e}^{-2 \lambda \phi_{m}^{j}} \mid \mathcal{F}_{j h}\right)+\frac{\left(\mathrm{e}^{-2 \lambda \phi_{m}^{j+1}}-\mathrm{e}^{-2 \lambda \phi_{m}^{j}}\right)}{h} \mathbb{E}\left(\hat{r}_{1 m}^{j+1} \mid \mathcal{F}_{j h}\right)\right] \mathrm{d} x \\
& -\mathbb{E} \sum_{j=0}^{m-1} \int_{G} \frac{\varrho}{\lambda^{4}} \frac{\left(\hat{r}_{2 m}^{j+1}-\hat{r}_{2 m}^{j}\right)}{h} \frac{\left(\mathrm{e}^{-2 \lambda \phi_{m}^{j+1}}-\mathrm{e}^{-2 \lambda \phi_{m}^{j}}\right)}{h} \hat{r}_{2 m}^{j+1} \mathrm{~d} x .
\end{aligned}
$$

By Hölder's inequality and the above equality, there is a positive constant $C=C(K, \lambda)$, independent of $m$, such that

$$
\begin{aligned}
& \mathbb{E} \sum_{j=0}^{m-1} \int_{G}\left\{\frac{\left(\hat{z}_{m}^{j+1}-\hat{z}_{m}^{j}\right)^{2}}{h^{2}} \mathrm{e}^{-2 \lambda \phi_{m}^{j}}+\frac{\varrho}{\lambda^{2}} \frac{\left[\mathbb{E}\left(\hat{r}_{1 m}^{j+1}-\hat{r}_{1 m}^{j} \mid \mathcal{F}_{j h}\right)\right]^{2}}{h^{2}} \mathrm{e}^{-2 \lambda \phi_{m}^{j}}\right. \\
& \left.+\frac{\varrho}{\lambda^{4}} \frac{\left(\hat{r}_{2 m}^{j+1}-\hat{r}_{2 m}^{j}\right)^{2}}{h^{2}} \mathrm{e}^{-2 \lambda \phi_{m}^{j}}+K \frac{\left(\hat{r}_{m}^{j+1}-\hat{r}_{m}^{j}\right)^{2}}{h^{2}}\right\} \mathrm{d} x \\
& \leq C \mathbb{E}\left[\sum_{j=1}^{m-1} \int_{G}\left(\left|\hat{z}_{m}^{j}\right|^{2}+\left|\hat{r}_{1 m}^{j}\right|^{2}+\left|\hat{r}_{2 m}^{j}\right|^{2}+K\left|\hat{r}_{m}^{j}\right|^{2}+\left|y_{m}^{j}\right|^{2}\right) \mathrm{d} x+\int_{G}\left|\hat{r}_{1 m}^{m}\right|^{2} \mathrm{~d} x\right] .
\end{aligned}
$$

Finally, by (A.12) and (4.10), recalling that $y \in L_{\mathbb{F}}^{2}\left(\Omega ; C\left([0, T] ; L^{2}(G)\right)\right)$, we get the desired estimate (4.11). This completes the Proof of Proposition 4.1.

\section{Appendix B. Proof of (5.6)}

This appendix is addressed to proving (5.6).

By (5.5), for a.e. $\omega \in \Omega, z_{\omega}=\tilde{z}(\omega) \in H^{1}\left(0, T ; L^{2}(G)\right)$ is a weak solution to the following random equation:

$$
\begin{cases}\mathcal{A} z_{\omega}=\tilde{r}_{1, \omega, t}+\tilde{r}_{2, \omega}+\lambda y_{\omega} \mathrm{e}^{2 \lambda \phi}+\check{r}_{\omega} & \text { in } Q, \\ z_{\omega}=0 & \text { on } \Sigma, \\ z_{\omega}(0)=z_{\omega}(T)=0 & \text { in } G .\end{cases}
$$

Here $\tilde{r}_{1, \omega, t}=\tilde{r}_{1, t}(\omega), \tilde{r}_{2, \omega}=\tilde{r}_{2}(\omega), y_{\omega}=y(\omega)$ and $\check{r}_{\omega}=\check{r}(\omega)$. Also, set $h_{\omega}=\tilde{r}_{1, \omega, t}+\tilde{r}_{2, \omega}+\lambda y_{\omega} \mathrm{e}^{2 \lambda \phi}+\check{r}_{\omega}$.

In the following, without loss of generality, we assume that $z_{\omega}$ is smooth and give a uniform estimate for it. Let $0<t_{1}<t_{2}<T$. Multiplying the first equation of (B.1) by $t^{2}(T-t)^{2} z_{\omega}$ and integrating it in $(0, T) \times G$, we get that

$$
\int_{t_{1}}^{t_{2}} \int_{G}\left|\nabla z_{\omega}\right|^{2} \mathrm{~d} x \mathrm{~d} t \leq C \int_{0}^{T} \int_{G}\left(\left|h_{\omega}\right|^{2}+\left|z_{\omega, t}\right|^{2}\right) \mathrm{d} x \mathrm{~d} t .
$$


Put

$$
E(t)=\frac{1}{2} \int_{G}\left(\left|z_{\omega, t}(t)\right|^{2}+\left|\nabla z_{\omega}(t)\right|^{2}\right) \mathrm{d} x .
$$

By the usual energy estimate for the first equation of (B.1) and noting the time reversibility of (B.1), we have that

$$
E(t) \leq C\left(E(s)+\int_{t_{1}}^{t_{2}} \int_{G}\left|h_{\omega}(\tau, x)\right|^{2} \mathrm{~d} x \mathrm{~d} \tau\right), \quad \forall t, s \in\left[t_{1}, t_{2}\right] .
$$

Integrating (B.3) with respect to $s$ from $t_{1}$ to $t_{2}$, we obtain

$$
E(t) \leq C\left(\int_{t_{1}}^{t_{2}} E(s) \mathrm{d} s+\int_{t_{1}}^{t_{2}} \int_{G}\left|h_{\omega}(\tau, x)\right|^{2} \mathrm{~d} x \mathrm{~d} \tau\right), \quad \forall t \in\left[t_{1}, t_{2}\right] .
$$

By (B.2) and (B.4), for any $t \in\left[t_{1}, t_{2}\right]$, there is a constant $C>0$ such that

$$
\left|z_{\omega, t}(t)\right|_{L^{2}(G)}^{2}+\left|z_{\omega}(t)\right|_{H_{0}^{1}(G)}^{2} \leq C \int_{0}^{T} \int_{G}\left(\left|h_{\omega}\right|^{2}+\left|z_{\omega, t}\right|^{2}\right) \mathrm{d} x \mathrm{~d} t .
$$

Applying the usual energy estimate to the first equation of (B.1) and noting the time reversibility of (B.1) again, similar to the proof of (B.3), we find that

$$
\left|z_{\omega}\right|_{C\left([0, T] ; H_{0}^{1}(G)\right) \cap C^{1}\left([0, T] ; L^{2}(G)\right)}^{2} \leq C\left(\left|z_{\omega, t}(t)\right|_{L^{2}(G)}^{2}+\left|z_{\omega}(t)\right|_{H_{0}^{1}(G)}^{2}+\left|h_{\omega}\right|_{L^{2}\left(0, T ; L^{2}(G)\right)}^{2}\right) .
$$

This, together with (B.5), implies that

$$
\left|z_{\omega}\right|_{C\left([0, T] ; H_{0}^{1}(G)\right) \cap C^{1}\left([0, T] ; L^{2}(G)\right)}^{2} \leq C \int_{0}^{T} \int_{G}\left(\left|h_{\omega}\right|^{2}+\left|z_{\omega, t}\right|^{2}\right) \mathrm{d} x \mathrm{~d} t .
$$

It follows that

$$
\mathbb{E}\left|z_{\omega}\right|_{\left.C\left([0, T] ; H_{0}^{1}(\Omega)\right)\right) \cap C^{1}\left([0, T] ; L^{2}(\Omega)\right)}^{2} \leq C \mathbb{E} \int_{0}^{T} \int_{G}\left(\left|h_{\omega}\right|^{2}+\left|z_{\omega, t}\right|^{2}\right) \mathrm{d} x \mathrm{~d} t .
$$

This, together with $\tilde{z} \in L_{\mathbb{F}}^{2}\left(G ; H^{1}\left(0, T ; L^{2}(G)\right)\right)$, implies that

$$
\tilde{z} \in L_{\mathbb{F}}^{2}\left(G ; C\left([0, T] ; H_{0}^{1}(G)\right)\right) \cap L_{\mathbb{F}}^{2}\left(G ; C^{1}\left([0, T] ; L^{2}(G)\right)\right) .
$$

\section{REFERENCES}

[1] C. Bardos, G. Lebeau and J. Rauch, Sharp sufficient conditions for the observation, control and stabilizion of waves from the boundary. SIAM J. Control Optim. 30 (1992) 1024-1065.

[2] J.-M. Coron, Control and Nonlinearity. Vol. 136 of Mathematical Surveys and Monographs. American Mathematical Society Providence, RI (2007).

[3] T. Duyckaerts, X. Zhang and E. Zuazua, On the optimality of the observability inequalities for parabolic and hyperbolic systems with potentials. Ann. Inst. Henri Poincaré, Anal. Non Lin. 25 (2008) 1-41.

[4] X. Fu, J. Yong and X. Zhang, Exact controllability for multidimensional semilinear hyperbolic equations. SIAM J. Control Optim. 46 (2007) 1578-1614.

[5] L. Hörmander, Linear Partial Differential Operators. Springer-Verlag, Berlin (1963).

[6] O.Yu. Imanuvilov, On Carleman estimates for hyperbolic equations. Asymptot. Anal. 32 (2002) 185-220.

[7] M.V. Klibanov, Carleman estimates for global uniqueness, stability and numerical methods for coefficient inverse problems. $J$. Inverse Ill-Posed Probl. 21 (2013) 477-560.

[8] M.M. Lavrent'ev, V.G. Romanov and S.P. Shishat.skiŭ, Ill-Posed Problems of Mathematical Physics and Analysis. Vol. 64 of Translations of Mathematical Monographs. American Mathematical Society, Providence, RI (1986).

[9] J.-L. Lions, Exact controllability, stabilization and perturbations for distributed systems. SIAM Rev. 30 (1988) 1-68.

[10] X. Liu, Global Carleman estimate for stochastic parabolic equaitons, and its application. ESAIM: COCV 20 (2014) 823-839.

[11] Y. Liu, Some sufficient conditions for the controllability of wave equations with variable coefficients. Acta Appl. Math. 128 (2013) 181-191. 
[12] Q. Lü, Observability estimate and state observation problems for stochastic hyperbolic equations. Inverse Probl. 29 (2013) 095011.

[13] Q. Lü and X. Zhang, Global uniqueness for an inverse stochastic hyperbolic problem with three unknowns. Commun. Pure Appl. Math. 68 (2015) 948-963.

[14] D. L. Russell, Controllability and stabilizability theory for linear partial differential equations: recent progress and open problems. SIAM Rev. 20 (1978) 639-739.

[15] X. Zhang, Explicit observability estimate for the wave equation with potential and its application. R. Soc. Lond. Proc., Ser. A, Math. Phys. Eng. Sci. 456 (2000) 1101-1115.

[16] X. Zhang, Carleman and observability estimates for stochastic wave equations. SIAM J. Math. Anal. 40 (2008) $851-868$.

[17] E. Zuazua, Exact controllability for semilinear wave equations in one space dimension. Ann. Inst. Henri Poincaré, Anal. Non Lin. 10 (1993) 109-129. 